\author{
RESEARCH ARTICLE \\ 10.1029/2018JC014803 \\ Key Points: \\ - The drag coefficient at the air-sea \\ interface follows Smith (1988) in \\ moderate winds and hardly \\ increases in light winds \\ - A constant imbalance term of 0.4 is \\ required in the inertial dissipation \\ flux calculation method, with data \\ sampled at a $1.5-\mathrm{m}$ height \\ - Stanton number estimates are \\ independent from wind speed in \\ moderate winds
}

Correspondence to:

D. Bourras,

denis.bourras@mio.osupytheas.fr

Citation:

Bourras, D., Cambra, R., Marié, L., Bouin, M.-N., Baggio, L., Branger, H., et al. (2019). Air-sea turbulent fluxes from a wave-following platform during six experiments at sea. Journal of Geophysical Research: Oceans, 124, 4290-4321. https://doi.org/10.1029/ 2018JC014803

Received 23 NOV 2018 Accepted 31 MAY 2019 Accepted article online 18 JUN 2019 Published online 26 JUN 2019

\section{Air-Sea Turbulent Fluxes From a Wave-Following Platform During Six Experiments at Sea}

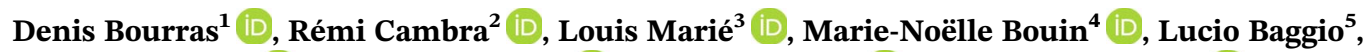

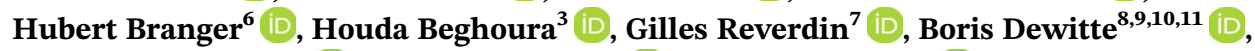 \\ Aurélien Paulmier ${ }^{12}$ (D) Christophe Maes $^{3}$ (D) Fabrice Ardhuin ${ }^{3}$ (D), Ivane Pairaud ${ }^{13,14}$, \\ Philippe Fraunié ${ }^{1}$, Christopher Luneau ${ }^{15}$, and Danièle Hauser ${ }^{5}$ iD
}

\begin{abstract}
${ }^{1}$ Aix Marseille Université, Université de Toulon, CNRS, IRD, MIO UM 110, Marseille, France, ${ }^{2}$ France Énergies Marines, Plouzané, France, ${ }^{3}$ LOPS, Plouzané IUEM Technopole Brest Iroise, Plouzané, France, ${ }^{4}$ Météo-France/CNRM, Plouzané, France, ${ }^{5}$ LATMOS, Guyancourt, CEDEX, France, ${ }^{6}$ Aix Marseille Université, CNRS, IRPHE, Ecole Centrale Marseille, Marseille, France, ${ }^{7}$ Sorbonne-Université, CNRS/IRD/DMNHN (LOCEAN), Paris, France, ${ }^{8}$ Centro de Estudios Avanzados en Zonas Áridas (CEAZA), Coquimbo, Chile, ${ }^{9}$ Millennium Nucleus for Ecology and Sustainable Management of Oceanic Island (ESMOI), Coquimbo, Chile, ${ }^{10}$ Departamento de Biología Marina, Universidad Católica del Norte (UCN), Coquimbo, Chile, ${ }^{11}$ Laboratoire d'Etudes en Géophysique et Océanographie Spatiales (LEGOS), Toulouse, France, ${ }^{12}$ LEGOS, IRD, CNRS, CNES, Université de Toulouse, Toulouse, France, ${ }^{13}$ Ifremer Méditerranée LERPAC, La Seyne sur Mer, France, ${ }^{14}$ Now at IFREMER, Université de Brest, CNRS UMR 6523, IRD, Laboratoire d'Océanographie Physique et Spatiale (LOPS), IUEM, Plouzané, France, ${ }^{15}$ OSU Institut Pythéas, CEREGE, Europôle Méditerranée, Aix en Provence, CEDEX 4, France
\end{abstract}

Abstract Turbulent fluxes at the air-sea interface are estimated with data collected in 2011 to 2017 with a low-profile platform during six experiments in four regions. The observations were carried out with moderate winds $(2-10 \mathrm{~m} / \mathrm{s})$ and averaged wave heights of $1.5 \mathrm{~m}$. Most of the time, there was a swell, with an averaged wave age (the ratio between wave phase speed and wind speed) being equal to $2.8 \pm 1.6$. Three flux calculation methods are used, namely, the eddy covariance (EC), the inertial dissipation (ID), and the bulk methods. For the EC method, a spectral technique is proposed to correct wind data from platform motion. A mean bias affecting the friction velocity $\left(u_{*}\right)$ is then evaluated. The comparison between EC $u_{*}$ and ID $u_{*}$ estimates suggests that a constant imbalance term $\left(\phi_{\mathrm{imb}}\right)$ equal to 0.4 is required in the ID method, possibly due to wave influence on our data. Overall, the confidence in the calculated $u_{*}$ estimates is found to be on the order of $10 \%$. The values of the drag coefficient $\left(C_{D}\right)$ are in good agreement with the parameterizations of Smith (1988, https://doi.org/10.1029/JC093iC12p15467) in medium-range winds and of Edson et al. (2013, https://doi.org/10.1175/JPO-D-12-0173.1) in light winds. According to our data, the inverse wave age varies linearly with wind speed, as in Edson et al. (2013, https://doi.org/10.1175/JPO-D-12-0173.1), but our estimates of the Charnock coefficient do not increase with wind speed, which is possibly related to sampling swell-dominated seas. We find that the Stanton number is independent from wind speed.

Plain Language Summary A small wave-following platform was deployed in 2011-2017 across four oceanic regions. The data are used to estimate turbulent fluxes, which are physical quantities that describe the exchanges of heat and momentum through the air-sea interface. In weather models, simplified representations of the fluxes are used, which themselves depend on coefficients named drag coefficient for momentum exchange and Stanton number for temperature exchange, respectively. In this study, we evaluate these coefficients. First, we compare the flux estimates from the three main available methods. We adjust the parameters in the methods to reach the best possible agreement between the calculated fluxes. Two types of corrections are proposed, depending on the method considered, because turbulence data are modified by the motion of the platform and by the proximity of waves. Data are corrected by applying a mean bias to the fluxes and by accounting for a nonmeasured term in the turbulence equations. Then, we analyze the wind dependence of the estimated drag coefficient and Stanton number. We find that drag is slowly increasing with wind speed, in agreement with existing models. Estimates of the Stanton number have biases but which do not depend on wind speed. 


\section{Introduction}

\subsection{Context of the Study}

Atmosphere, ocean, and waves are coupled through turbulent energy fluxes across the air-sea interface. The fluxes considered in this paper are the momentum flux $\tau / \rho$, where $\rho$ is the density of air (in $\mathrm{kg} / \mathrm{m}^{3}$ ), $\tau$ is the wind stress (in $\mathrm{N} / \mathrm{m}^{2}$ ), and $H_{S}$ is the sensible heat flux (in $\mathrm{W} / \mathrm{m}^{2}$ ). In numerical models that predict waterwave fields or circulations of the ocean or the atmosphere, the fluxes are generally parameterized using the Monin-Obukhov (MO hereafter) similarity theory, which may be summarized as

$$
\begin{gathered}
\tau / \rho=C_{D} U^{2}=u *^{2} \\
H_{S} / \rho C_{P}=C_{H} U \Delta T
\end{gathered}
$$

where $u_{*}$ is the friction velocity (in $\mathrm{m} / \mathrm{s}$ ), $U$ is the mean wind speed relative to the sea surface (in $\mathrm{m} / \mathrm{s}$ ), defined here as the norm of the averaged values of the horizontal components of the wind vector, $\Delta T$ is the mean temperature difference between sea and air (in $\mathrm{K}$ ), $C_{P}$ is the specific heat of air (in $\mathrm{J} \cdot \mathrm{kg} \cdot \mathrm{K}$ ), and $C_{D}$ and $C_{H}$ are the so-called turbulent exchange coefficients, referred to as drag coefficient and Stanton number, respectively. The exchange coefficients may be taken as constant or they may be parameterized as a function of wind speed, of the stability of the Surface Atmospheric Boundary Layer (SABL), or of sea wave characteristics. Thus, the widely used notion of flux parameterization, in fact, always refers to the parameterization of the exchange coefficients.

As air-sea fluxes are a boundary condition in numerical models, the choice of one rather than another parameterization may strongly condition the model output fields. Recently, Brodeau et al. (2017) compared wind stress estimates calculated with two known flux algorithms at global scale over a 30-year period. They found a discrepancy of $10 \%$. They conclude that there is a strong need for further validation of the flux algorithms with respect to observations. Converging toward a more universally accepted parameterization of the Charnock coefficient (Charnock, 1955) or the drag coefficient on wind speed remains a key priority. This illustrates well the expectations of modelers in this field. In the case of wind stress, however, the value of $10 \%$ corresponds to the claimed accuracy of the known COARE 3.0 bulk algorithm (Fairall et al., 2003) for wind speeds between 10 and $20 \mathrm{~m} / \mathrm{s}$. Therefore, it may just be the state of the art, in addition to the fact that $10 \%$ may already be considered as accurate from the point of view of scientists who struggle with noisy data. Nevertheless, the conclusions of Brodeau et al. (2017) suggest that (1) some aspects of surface fluxes parameterizations are yet to be explored and that (2) more accurate validation data are required.

In this paper, we take advantage of data collected over 7 years with a novel wave-following platform dedicated to air-sea flux measurements (Bourras et al., 2014, B14 hereafter) to contribute to surface flux parameterization, with the following specificities: our data were collected in the open sea, mostly in conditions of swell, with significant wave heights $h s w$ averaging to $1.5 \mathrm{~m}$, and in light to moderate wind conditions, that is, $2 \leq U \leq 10 \mathrm{~m} / \mathrm{s}$. The turbulence measurements were performed at a $1.5-\mathrm{m}$ height, which is in a part of the atmospheric layer influenced by the presence sea waves (e.g., B14; Hara \& Sullivan, 2015). These turbulence data are restricted to the wind stress and to the buoyancy flux $H_{S V}$, which is the virtual (noncorrected from humidity) counterpart of $H_{S}$, because no instrument was available on the platform to calculate the moisture flux for most of the data. Finally, in this study, the turbulent fluxes are estimated with three methods: the bulk method (COARE 3.0), the eddy covariance (EC) method (e.g., Pedreros et al., 2003), and the inertial dissipation (ID) method (e.g., Yelland et al., 1994).

Hereafter in this introduction, we first review the science issues that shall be addressed with our data, namely, the relation between wind stress and waves, the parameterization of drag in light- to medium-range wind conditions, and the parameterization the turbulent exchanges of temperature between air and sea. Second, we provide a review on two issues associated with flux estimation, as the methodology strongly conditions the accuracy of the calculated fluxes: the platform motion correction with the EC flux calculation method and the evaluation of the imbalance term in the ID method.

\subsection{Surface Flux Parameterizations Issues}

\subsubsection{Wind Stress Versus Wave State}

The relation between wind stress and wave state was addressed in a number of papers (e.g., Donelan et al., 1997). The existing parameterizations of surface drag depend on wave slope or wave age (e.g., Drennan et al., 
2003; Oost et al., 2002; Taylor \& Yelland, 2001). However, according to Edson et al. (2013), the parameterization of the Charnock coefficient as a function of wind speed only is sufficient to estimate wind stress over the open ocean, without using wave characteristics except in specific cases such as light wind and swell, for example. If true, this is fortunate, because accurate wave characteristics are not available in a number of applications. According to Edson et al. (2013), the parameterizations of the wind stress as a function of wind speed, wave age, and wave slope are equivalent, because the inverse wave age varies nearly linearly with wind speed. This needs to be confirmed as it may be considered as an oversimplification of the effect of waves on air-sea momentum transfer.

\subsubsection{Turbulent Drag in Light Winds}

According to Edson et al. (2013), at wind speeds smaller than $4 \mathrm{~m} / \mathrm{s}$, swell travelling faster than wind speed would reduce the drag, thus generating a low-level jet, a hypothesis that was recently supported by numerical simulations (Sullivan et al., 2008). There is a growing consensus that wind-swell interactions reduce the drag at low wind speeds (e.g., Hanley et al., 2010). Evaluating $C_{D}$ in these conditions is possibly an ill-posed problem, which is supported by the large scatter of drag coefficient estimates reported in swell-dominated conditions (Vickers et al., 2013). In apparent contrast, Potter (2015) analyzed the drag coefficient as a function of the ratio between swell energy and wind energy. His low wind data $\left(U_{10 N}<4 \mathrm{~m} / \mathrm{s}\right.$, with $U_{10 N}$ the wind speed referenced at $z=10 \mathrm{~m}$ in neutral stability conditions) clearly show an increase of the drag coefficient that is not much commented because the study was more focused on drag at larger wind speeds. Potter (2015) argued that the observed increase in $C_{D}$ was possibly due to wind gustiness inducing departure from the MO theory, combined with increased viscous effects. Nevertheless, the data reported by Garcia-Nava et al. (2012) and Ocampo-Torres et al. (2011) show even better the increased drag coefficient effect at low winds. There, it is attributed to the so-called swell-induced stress, which is assumed to depend on swell steepness and on the wave age. The increase of fluxes may also be due to an upward momentum flux when significant swell is travelling faster than the wind (Grachev \& Fairall, 2001; Hanley et al., 2010). Aircraft data recorded at an altitude of $35 \mathrm{~m}$ also revealed a $C_{D}$ increase at low winds (Kalogiros \& Wang, 2011). A similar increase of $C_{D}$ at low winds is also noticed over lakes by Sahlée et al. (2014), Figure 11). In addition, the different studies still debate whether there is a need for parameterizing the drag coefficient as a function of wind only or wind plus sea state (e.g., Andreas et al., 2012) and whether $C_{D}$ would increase or not in swelldominated conditions, as interactions more complex may occur at the interface, according to Högström et al. (2013). At low wind speeds, the behavior of the turbulent drag is therefore still a question to explore.

1.2.3. Stanton Number and Wind Speed

Historical and acknowledged values of the Stanton number for moderate wind conditions are $1.13 \times 10^{-3}$ if the surface layer is unstable and $0.66 \times 10^{-3}$ under stable conditions (Large \& Pond, 1982), so that the Stanton number is expected to be smaller in stable conditions than in unstable conditions. The confidence intervals of experimental estimates of the Stanton number are large, in the range $0.5 \times 10^{-3}-1.5 \times 10^{-3}$, according to the HEXOS (DeCosmo et al., 1996) and CBLAST (Zhang et al., 2008) reference experiments. The most recent studies point out the lack of understanding in this field, that is, the discrepancy between theory and observations, specifically at large wind speeds (Mueller \& Veron, 2010). According to these authors, the coefficient slowly increases with wind speed at large wind speed values. However, for wind values smaller than $10 \mathrm{~m} / \mathrm{s}$, the coefficient may increase or decrease, depending on stratification (e.g., Figure 1b in Mueller \& Veron, 2010). This contrasts with earlier findings of Makin and Mastenbroek (1996) who suggested that the Stanton number was rather independent of wind speed in the range 4-10 m/s. Larsen et al. (2004) and more recently Smedman et al. (2007) reached a similar conclusion. Specifically, the later authors found that in unstable conditions, $C_{H}$ was independent of wind speed, if the wind speed was below $9 \mathrm{~m} / \mathrm{s}$ and if the mean sea minus air temperature difference was larger than $3^{\circ}$. For larger wind values, the same authors found that $C_{H}$ gradually increases with wind speed.

\subsection{Experimental Issues With Flux Calculation \\ 1.3.1. Motion Correction in the EC Method}

The EC method is the application of the canonical flux definition, which is written as 


$$
\begin{gathered}
\tau / \rho=\left({\overline{u^{\prime} w^{\prime}}}^{2}+{\overline{v^{\prime} w^{\prime}}}^{2}\right)^{1 / 2} \\
H_{S} / \rho C_{P}=-\overline{w^{\prime} \theta^{\prime}}
\end{gathered}
$$

where $u, v$, and $w$ are the wind vector components with $w$ along the vertical and positive upward, $\theta$ is the air potential temperature, and the over lines and quotes denote mean and fluctuating parts of wind or temperature after applying Reynolds decomposition.

The application of equations (3) and (4) to sea wind data would lead to biased estimates because wind data are contaminated by platform motion (for ships and buoys) and flow distortion, mostly along the vertical (e.g., Edson et al., 1998; Kaimal \& Finnigan, 1994; Miller et al., 2008; Pedreros et al., 2003). This implies that a correction of $w^{\prime}$ is required. A common correction process consists in subtracting the vertical velocity of the platform from $w^{\prime}$ (Pan et al., 2005). However, among others, Bourras et al. (2009, B09 hereafter) and Prytherch et al. (2015) suspected that only part of the vertical motion is converted into vertical relative wind. In addition, a phase shift between the platform vertical motion and the vertical wind measured can occur because of the disturbing effect of the platform body at turbulent scales. This implies that one needs to account not only for mean flow distortion but also for the flow distortion at turbulent scales.

The correction proposed by Prytherch et al. (2015) involves a slightly more improved subtraction with coefficients inferred from a regression between data and the expected Kaimal et al. (1972) bell-shaped cospectra obtained over land 50 years ago. Indeed, they show that the subtraction procedure removes the platform motion peak in their cospectra and produces flux data with less scatter. However, this comes at the cost of a strong assumption on the shape of the cospectra and of a very restrictive data selection process. Our understanding is that platform motion correction is required at turbulent scales but that its application is still an issue that needs to be further addressed.

\subsubsection{Imbalance Term in the ID Method}

The ID method is a two-step method that begins with the calculation of the dissipation rate of turbulence $\varepsilon$. According to the Kolmogorov cascade theory and after applying the hypothesis of Taylor's frozen turbulence in order to convert wave numbers to frequencies, $\varepsilon$ may be written as

$$
\varepsilon=\frac{2 \pi}{U_{r}}\left(\frac{\left|E_{U}(f)\right|^{2}}{c}\right)^{\frac{3}{2}} f^{\frac{5}{2}}
$$

where $c$ is the Kolmogorov constant (0.55), $U_{r}$ is the module of the wind vector relative to the measurement platform (the apparent wind), $f$ is the frequency in $\mathrm{Hz}$, and $\left|E_{U}(f)\right|^{2}$ is the frequency power spectrum of $u$ in the inertial subrange that is in a spectral region where energy decreases as $f^{-\frac{5}{3}}$, which usually occurs between 1 and $10 \mathrm{~Hz}$ with our data.

In a second step, $u_{*}$ is deduced from $\varepsilon$ through the diagnostic equation of Turbulent Kinetic Energy (TKE; Stull, 1988). Under the classical hypotheses of horizontal homogeneity and steadiness of the flow associated with the application of the MO similarity theory to the SABL, the TKE equation is written as

$$
u_{*}=(\kappa z \varepsilon)^{\frac{1}{3}}\left(\phi_{U}\left(\frac{z}{L}\right)-\frac{z}{L}-\phi_{\mathrm{imb}}\right)^{-\frac{1}{3}}
$$

where $\kappa$ is the Von Karman constant (0.4), $z$ is the height of measurement in meters, $\phi_{U}$ is the Businger-Dyer relationship, $\phi_{\mathrm{imb}}$ is the so-called imbalance term, and $\frac{z}{L}$ is the MO ratio that defines dynamic stability of the SABL, which is written as

$$
\frac{z}{L}=-\kappa z\left(g / \overline{\theta_{V}}\right) \overline{w^{\prime} \theta_{V}^{\prime}} / u_{*}^{3}
$$

where $g$ is gravity acceleration in $\mathrm{m} / \mathrm{s}^{2}, \theta_{V}=\theta(1+0.61 q)$ is the virtual potential temperature, and $\overline{w^{\prime} \theta_{V}}$ is the buoyancy flux, which is used in this paper as

$$
H_{S V} / \rho C_{P}=-\overline{w^{\prime} \theta_{V}^{\prime}}
$$


In equation (6), $\phi_{\mathrm{imb}}$ is a residual term that is the sum of two terms: the TKE turbulent transport term and the pressure correlation term (Stull, 1988). In practice, the pressure correlation term at the air-sea interface is not yet easy to measure with the available instruments. From the point of view of pure turbulence, it originates from the process of return to isotropy, but close to the waves, that is, typically below a height of $3 \mathrm{~m}$, the pressure term is modified because of the presence of the wavefield. With the classical Reynolds decomposition of turbulent variables, the definition of the pressure term is the divergence of $\overline{w^{\prime} p^{\prime}}$, where $p^{\prime}$ corresponds to the static pressure fluctuations. However, many studies (e.g., CifuentesLorenzen et al., 2018; Edson \& Fairall, 1998; Hara \& Sullivan, 2015) use a three-scale decomposition in which the wave-related fluctuations are denoted with a tilde, and the wave-related wind-pressure covariance is referred to as $\overline{\widetilde{w}} \widetilde{p}$. Cifuentes-Lorenzen et al. (2018) successfully managed to indirectly estimate this term by evaluating momentum and energy budgets at the interface deduced from measurements of wave spectra and wind turbulence at one altitude. Their estimates of $\widetilde{w \widetilde{p}^{-}}$range from to 0.5 to $5 \mathrm{~W} / \mathrm{m}^{2}$ in condition of sea waves driven by a strong wind input. This is unfortunately not conditions of swelldominated seas that were present in our study.

With experimental data, an advantage of the ID flux calculation method is that it is not affected by platform motion contrary to the EC method. However, parameters need adjustments to produce accurate estimates, and since the late 1990s, there is a controversy on these adjustments (e.g., Dupuis et al., 1997; Taylor \& Yelland, 2000). For some authors, the value of $c$ could be modified and $\phi_{\text {imb }}$ should be neglected, while others suggested adjusting $\phi_{\mathrm{imb}}$ without changing $c$. For example, B09 found that -0.46 $\frac{z}{L}$ was an appropriate parameterization of $\phi_{\text {imb }}$ with data collected on a large research vessel, which was in good agreement with Dupuis et al. (1997) results, that is, $-0.65 \frac{z}{L}$ under unstable conditions. B14 found that the same parameterization was also adapted to the small number of data of the FROMVAR 2011 experiment. With a larger data set further detailed in the present paper, Cambra (2015) rather found that a constant imbalance term on the order of 0.5 should be accounted for, whereas the parameterization in $\frac{z}{L}$ had little impact on estimating friction velocity with the ID method. Interestingly, $\phi_{\mathrm{imb}}=0.5$ compares well to the minimum of the dimensionless turbulence dissipation rate estimated as 0.7 by Edson and Fairall (1998). Therefore, the dimensionless dissipation rate of turbulence should be further investigated.

In addition, according to the large eddy simulations of Hara and Sullivan (2015) and to the recent results of Cifuentes-Lorenzen et al., (2018), the dissipation rate and the turbulent part of the momentum flux should decrease close to a wavefield compared to their wave-induced counterparts, partly because of the wave pressure effect. Therefore, we need to revisit the estimation of $\phi_{\mathrm{imb}}$, the value of which is likely height dependent and may be specifically modified for our wave-follower data. Indeed, most of our measurements are taken close to the surface, that is, at $1.5 \mathrm{~m}$, which may be either considered to be in the midpart of the wave boundary layer (WBL) according to the rough estimation of 1-3 m given by Cifuentes-Lorenzen et al. (2018) or to be in well inside the WBL according to the definition of Hara and Sullivan (2015), that is, at normalized heights $k \zeta$ (in wave-following coordinates) ranging from 0.06 to 0.13 , which is small compared to their WBL height that peaks at $k \zeta \sim 0.7$ in their simulations.

\subsection{Overview}

The paper is organized as follows. In section 2, we present platform and instruments and the sea experiments and encountered environmental conditions. The last two subsections of section 2 are devoted to the processing of other data than turbulence. First, we document the wave characteristics encountered during the experiments such as significant wave height or wave age, the calculation of which-based on platform motion data-is specific to our platform. Next, we present a new spectral motion correction method for vertical wind speed and we verify whether it performs better than the usual correction based on a subtraction between vertical wind and vertical velocity of the platform.

In section 3, we present a classical analysis of the spectral properties of turbulence, with power spectra and cospectra. Specifically, we identify the inertial subrange, and we compare the turbulence cospectra to the reference cospectra of Kaimal et al. (1972), to evaluate the performance of the correction method. In a following section, we calculate dissipation rates, we identify the most appropriate method to achieve it, and we estimate the Kolmogorov constant. 

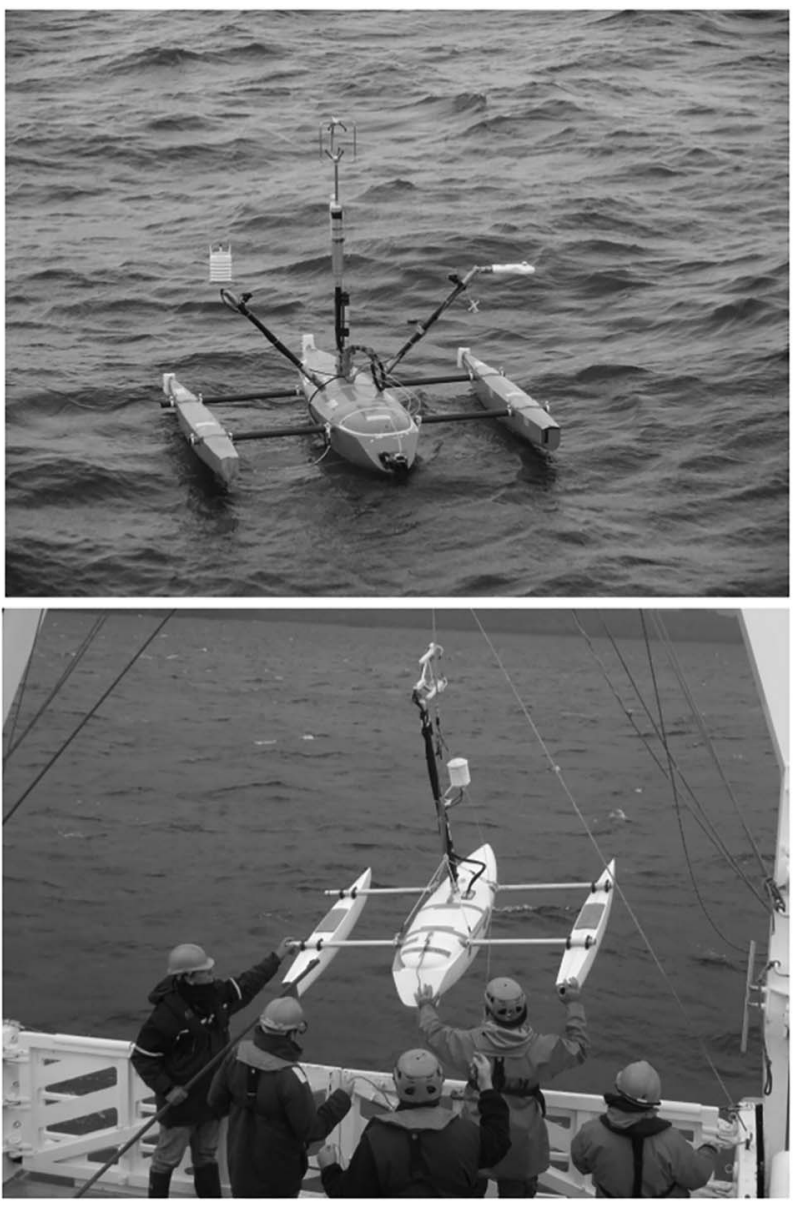

Figure 1. Pictures of the two OCARINA prototypes, referred to as OCARINA \#1 (earlier version, upper panel) and OCARINA \#2 (more recent test version, lower panel).
In section 4, we compare the outputs of the three flux calculation methods, namely, the bulk method, the EC method (with and without correction of vertical platform motion), and the ID method, and we analyze the discrepancies. This raw comparison suggests whether and how motion correction should be applied. Once the EC $u_{*}$ estimates are adequately corrected, we adjust the parameters in the ID method. Specifically, we verify whether it is more appropriate to use an imbalance term than modifying $c$ in the ID method, and we give an estimate of the dimensionless dissipation rate of turbulence. Next, we evaluate the drag coefficient, proposing a parameterization of the drag as a function of wind speed, and we analyze the relations between the drag coefficient, the Charnock coefficient, and wave characteristics. Last, we analyze how the Stanton number depends on wind speed and stability.

In section 5, we attempt to identify whether or not the imbalance term found is related to the proximity of sea waves, and we check if it is overall more efficient to apply the spectral motion correction to individual data rather than using it to evaluate a mean correction of the EC $u_{*}$ estimates. Conclusions follow in section 6 .

\section{Platform, Sea Experiments, and Data Processing}

\subsection{Platforms}

The OCARINA platform was already thoroughly described in B14. A second prototype was built since then, so we recall here the main features of the platforms and their respective instrumental setup. In the following, the two prototypes are referred to as \#1 (earlier version) and \#2 (recent version), respectively. Pictures of the two prototypes are shown in Figure 1, and the list of available instruments and associated measured variables and the placement heights of the sensors in each prototype are summarized in Table 1.

OCARINA \#1 was equipped with a Gill R3-50 3-D sonic anemometer that measured $u^{\prime}, v^{\prime}, w^{\prime}$, and the sonic temperature, which was converted into virtual temperature $\theta_{V}$ (the two variables shall be confused hereafter because the conversion that was applied was on the order of a thousandth of a degree for the temperature fluctuations), one Vaisala WXT-520 meteorological station that measured air temperature $(T)$, relative humidity $(\mathrm{RH})$, and atmospheric pressure $(P)$, one Campbell CNR4 radiation flux sensor that measured upwelling and downwelling solar and longwave radiation fluxes, and one Cadden MTI-G inertial sensor that measured attitude angles and linear velocities along three axes at $50 \mathrm{~Hz}$. Last, there was a seabird SBE-37SI sensor under it for measuring the Sea Surface Temperature (SST).

OCARINA \#2 was equipped with a Campbell Irgason EC100 that combined sonic anemometer with a fast humidity and $\mathrm{CO}_{2}$ sensors. Meteorological data were sensed by a Rotronic HC2S3 sensor (for $T$ and $\mathrm{RH}$ ) and one Vaisala PTB-110 atmospheric pressure sensor. There was one sea surface sensor, namely, a 100 Ohms platinum resistor for SST. Motion was recorded by a NAVEOL NAV-01H nine-axis motion sensor. The EC100 used on OCARINA \#2 is a promising instrument that gathers several turbulence sensors in a compact shape. However, this comes at the cost of a bulky appearance that distorts the air flow, affecting somehow the mean wind and the vertical momentum flux (Horst et al., 2016). Both of them either increase or decrease depending on the horizontal and vertical wind angles. Horst et al. (2016) showed that the distortion effect was not symmetric, which means that data correction is even trickier because it depends on whether the flow comes from the left side or from the right side of the instrument. We did not apply any correction to Irgason data because, due to a lack of experience from our part, the sensor was systematically placed at a $90^{\circ}$ angle from the mean flow direction, a setting for which the correction is not well documented in Horst et al. (2016). Please note finally that no surface current meter is available on the platforms. 
Table 1

Instruments Installed on the Two OCARINA Prototypes

\begin{tabular}{|c|c|c|c|c|}
\hline \multicolumn{5}{|l|}{ OCARINA \#1 } \\
\hline Measured quantities & Variable names (units) & Sampling rate $(\mathrm{Hz})$ & Instrument & Height (m) \\
\hline Wind vector & $u, v, w(\mathrm{~m} / \mathrm{s})$ & 50 & Gill R3-50 & $1.5-1.6$ \\
\hline Speed of sound & $c(\mathrm{~m} / \mathrm{s})$ & 50 & - & $1.5-1.6$ \\
\hline Air temperature & $T\left({ }^{\circ} \mathrm{C}\right)$ & 1 & Vaisala WXT-520 & $0.8-0.9$ \\
\hline Relative humidity & RH $(\%)$ & 1 & - & $0.8-0.9$ \\
\hline Atmospheric pressure & Patm (hPa) & 1 & - & $0.8-0.9$ \\
\hline $\begin{array}{l}\text { Solar and infrared radiation fluxes, } \\
\text { upward and downward }\end{array}$ & Fsol_dn, Fsol_up, Fir_dn, and Fir_up $\left(\mathrm{W} / \mathrm{m}^{2}\right)$ & 1 & Campbell CNR4 & $0.7-0.8$ \\
\hline Time, position, and motion & $\begin{array}{l}\text { time, lon }\left(^{\circ}\right), \text { lat }\left({ }^{\circ}\right), \text { lin_acc_xyz }\left(\mathrm{m} / \mathrm{s}^{2}\right) \text {, } \\
\text { and ang_vel_xyz (rad/s) }\end{array}$ & 50 & Xsens MTI-G & 0.1 \\
\hline SST and salinity & $\operatorname{SST}\left({ }^{\circ} \mathrm{C}\right)$ and SSS (psu) & 1 & Seabird SBE-37SI & $-0.1-0.3$ \\
\hline \multicolumn{5}{|l|}{ OCARINA \#2 } \\
\hline Wind vector & $u, v, w(\mathrm{~m} / \mathrm{s})$ & 25 & Campbell Irgason & 1.6 \\
\hline Air temperature & $T\left({ }^{\circ} \mathrm{C}\right)$ & 25 & - & 1.6 \\
\hline Specific humidity & $q(\mathrm{~g} / \mathrm{kg})$ & 25 & - & 1.6 \\
\hline Air temperature & $T\left({ }^{\circ} \mathrm{C}\right)$ & 1 & Rotronic $\mathrm{HC} 2 \mathrm{~S} 3$ & 1 \\
\hline Relative humidity & RH (\%) & 1 & - & 1 \\
\hline Atmospheric pressure & Patm $(\mathrm{hPa})$ & 1 & Vaisala PTB-110 & 1 \\
\hline Time and position & time, lon $\left(^{\circ}\right)$, lat $\left(^{\circ}\right)$ & 1 & Sirf EM506 GPS & 0. \\
\hline Motion & lin_acc_xyz (m/s $\left.\mathrm{s}^{2}\right)$, ang_vel_xyz (rad/s) & 25 & Naveol NAV01H & 0. \\
\hline SST & SST $\left({ }^{\circ} \mathrm{C}\right)$ & 1 & PT100 platinum temperature sensor & -0.3 \\
\hline
\end{tabular}

Therefore, $U$ is hereafter approximated by its value with respect to the seafloor (i.e., it is corrected with GPS and motion data), not with respect to the sea surface velocity.

\subsection{Sea Experiments}

OCARINA was deployed in the framework of five oceanographic campaigns over 7 years, namely, FROMVAR 2011, STRASSE 2012, AMOP 2014, BBWAVES2015, BBWAVES 2016, and UPCAST 2017. As can be inferred from Figure 2 and Table 2, OCARINA was tested in four environments, namely, the tropical Atlantic (STRASSE 2012), the southern eastern Pacific upwelling region (AMOP 2014), off the Brittany coast (BBWAVES), and in a western coastal Mediterranean area (UPCAST 2017).

OCARINA prototype \#1 was deployed in all experiments, whereas prototype \#2 was only deployed during BBWAVES 2015. Therefore, in the following, the different data sets are named as a function of the name and year of the experiment and as a function of the prototype number (indicated only if it is prototype \#2). This results in seven data sets that are hereafter referred to as F11, S12, A14, B15, B15-2, B16, and U17 (Table 2).

A total of 57 days were spent at sea, with the OCARINA acquisition systems powered on during 20 days (467 $\mathrm{hr}$ ), and after application of quality tests and supervision of data, 13.5 days (324 hr) of data were eventually retained. The most statistically represented experiments are S12 (77 hr) and A14 (181 hr). OCARINA drift velocity averaged between 0.4 and $0.7 \mathrm{~m} / \mathrm{s}$, and the standard deviations of the roll and pitch angles averaged between $0.2^{\circ}$ and $6.8^{\circ}$, with larger maximum angles for B15-2 data $\left(25^{\circ}\right)$ than for the other experiments $\left(16^{\circ}\right)$, the head of the EC100 at the top of the central mast being heavier $(2.8 \mathrm{~kg})$ than the head of the R3-50 anemometer $(1 \mathrm{~kg})$.

Data were collected in conditions of $T$ and SST in the range $13-28{ }^{\circ} \mathrm{C}$ and with specific humidity values ranging from 6 to $18 \mathrm{~g} / \mathrm{kg}$. Stability conditions in the SABL may be described in terms of MO ratio. Hereafter, we use the bulk estimates of $\frac{z}{L}$ to identify stability conditions, because our EC and ID estimates of $z / L$ have more scatter (not shown). The median $\frac{z}{L}$ values range from -0.03 to 0.00 , which means that the SABL is close to neutrality in our data (Table 2). For A14, the SABL is neutral but on the stable side, as the median value of $\frac{z}{L}$ equals -0.03 and as the maximum value of $\frac{z}{L}$ exceeds 0.1 . However, the SABL is not significantly stable for A14, because the rms deviation of $\frac{z}{L}$ is small, that is, 0.02 . For S12, the SABL is 

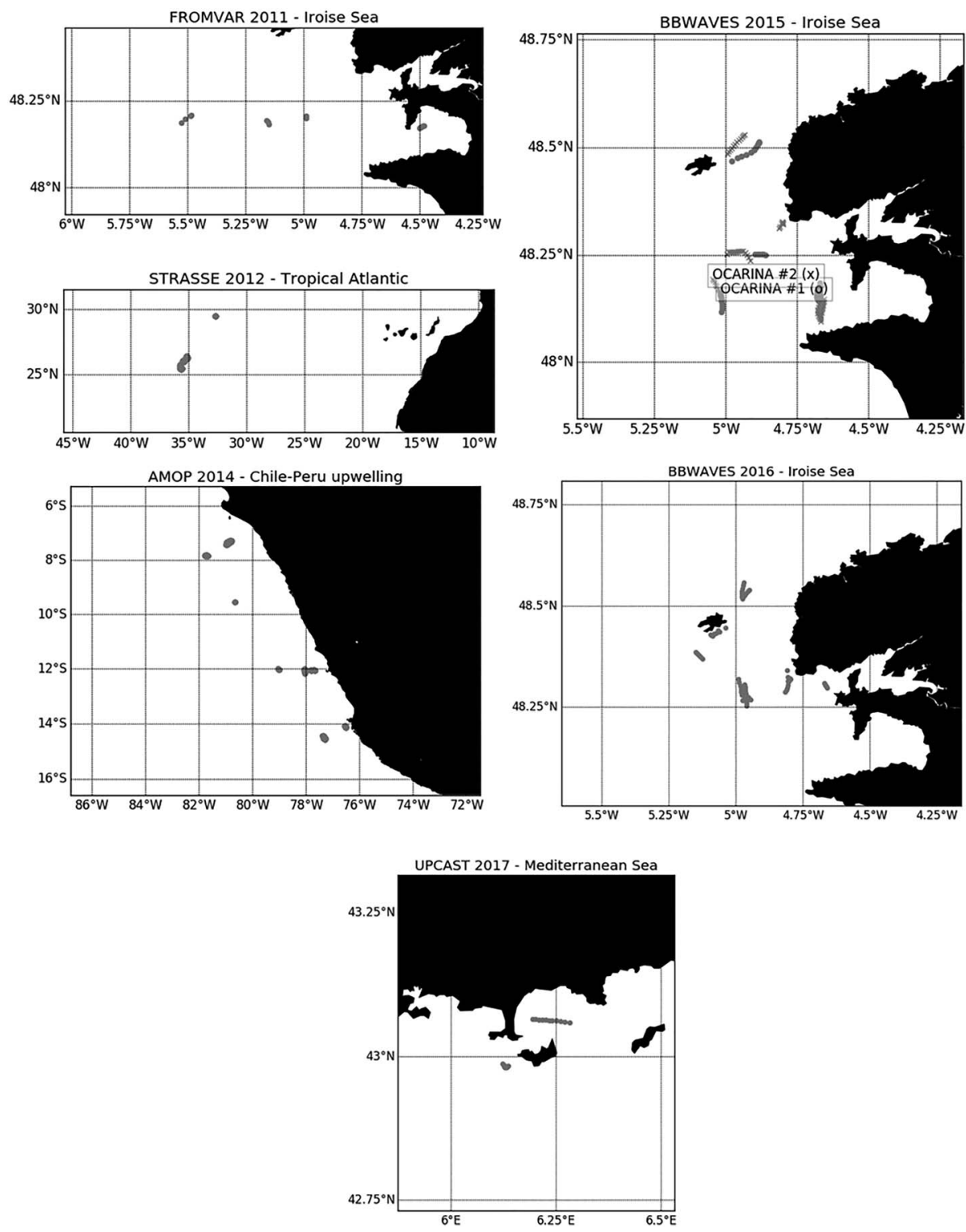

Figure 2. Location and years of deployment of the platforms.

neutral in average and it is unstable by episodes. Note, however, that there are no free convection events (defined as $\frac{z}{L} \ll-1$ ) as the minimum value of $\frac{z}{L}$ is -0.57 .

\subsection{Sea Wave Conditions}

The significant wave height estimates (hsw) and the period of most significant waves (Tsw) were inferred from inertial platform output data, which are vertical acceleration ( $a c c z)$ for B15-2 and vertical velocity (velz) for the other campaigns.

Significant wave height estimates $(h s w)$ were calculated as 4 times the integral value of the spectra of surface elevation $(\eta)$, between 1 and $30 \mathrm{~s}$ in periods. Time series of $\eta$ were themselves obtained by applying a double (single) spectral integration to the recorded time series of $a c c z$ (velz). The resulting estimates of $h s w$ average $1.5 \pm 0.4 \mathrm{~m}$. Individual daily average values per experiment range from 0.6 to $2 \mathrm{~m}$, as reported in Table 2 .

The most significant wave period ( $T s w$, or period of dominant waves) estimation technique slightly evolved since B14, in which the location of the largest peak was detected in the surface elevation $(\eta)$ spectrum. With the availability of five new data sets, a wider range of wave conditions was observed and we noticed that the 
Table 2

Summary of the Conditions Encountered During the Experiments

\begin{tabular}{|c|c|c|c|c|c|c|c|c|}
\hline \multicolumn{2}{|l|}{ Experiment } & F11 & S12 & A14 & B15 & B15-2 & B16 & U17 \\
\hline \multicolumn{2}{|l|}{ Year } & 2011 & 2012 & 2014 & 2015 & 2015 & 2016 & 2017 \\
\hline \multicolumn{2}{|l|}{ Prototype } & $\# 1$ & $\# 1$ & $\# 1$ & $\# 1$ & \#2 & $\# 1$ & $\# 1$ \\
\hline \multicolumn{2}{|l|}{ Sea campaign } & FROMVAR & STRASSE & AMOP & BBWAVES & BBWAVES & BBWAVES & UPCAST \\
\hline \multicolumn{2}{|c|}{ Location time at sea/useful data (hr) } & Iroise sea & Tropical Atlantic & Chile-Peru & Iroise Sea & Iroise Sea & Iroise Sea & Mediterranean \\
\hline \multirow[t]{4}{*}{ Wind speed $(\mathrm{m} / \mathrm{s})$} & $\min$ & 1.2 & 1.4 & 1.7 & 4.2 & 3.7 & 1.4 & 2.6 \\
\hline & $\bar{x}$ & 4.9 & 4.7 & 4.8 & 6.1 & 6.4 & 4.4 & 6.5 \\
\hline & $\max$ & 8.6 & 7.6 & 8.8 & 7.7 & 10.4 & 8.5 & 10.4 \\
\hline & $\sigma$ & 2.6 & 1.4 & 1.6 & 1.1 & 1.5 & 2.0 & 2.8 \\
\hline \multirow[t]{4}{*}{ Air temperature $\left({ }^{\circ} \mathrm{C}\right)$} & $\min$ & 15.1 & 25.6 & 17.5 & 13.3 & 13.0 & 14.3 & 18.5 \\
\hline & $\bar{x}$ & 16.4 & 26.4 & 22.0 & 14.7 & 14.8 & 15.8 & 19.1 \\
\hline & $\max$ & 17.6 & 27.1 & 24.6 & 16.0 & 16.3 & 18.1 & 19.5 \\
\hline & $\sigma$ & 1.0 & 0.3 & 1.7 & 0.7 & 0.7 & 0.8 & 0.3 \\
\hline \multirow[t]{4}{*}{$\operatorname{SST}\left({ }^{\circ} \mathrm{C}\right)$} & $\min$ & 14.6 & 26.9 & 16.5 & 14.3 & 14.3 & 14.0 & 17.7 \\
\hline & $\bar{x}$ & 16.2 & 27.2 & 21.8 & 15.0 & 14.9 & 15.1 & 18.3 \\
\hline & $\max$ & 17.0 & 27.6 & 25.3 & 15.4 & 15.3 & 16.8 & 18.8 \\
\hline & $\sigma$ & 0.8 & 0.2 & 2.2 & 0.4 & 0.3 & 0.8 & 0.3 \\
\hline \multirow{4}{*}{$\frac{z}{L}$} & $\min$ & -0.15 & -0.57 & -0.05 & -0.07 & -0.17 & -0.10 & 0.00 \\
\hline & med & 0.01 & -0.03 & 0.00 & -0.01 & -0.01 & 0.01 & 0.00 \\
\hline & $\max$ & 0.73 & -0.01 & 0.12 & 0.01 & 0.02 & 0.07 & 0.07 \\
\hline & $\sigma$ & 0.19 & 0.09 & 0.02 & 0.02 & 0.03 & 0.03 & 0.02 \\
\hline \multirow[t]{4}{*}{ Specific humidity (g/kg) } & $\min$ & 8.0 & 13.3 & 10.8 & 6.4 & 7.3 & 7.1 & 7.7 \\
\hline & $\bar{x}$ & 9.5 & 15.8 & 14.4 & 7.4 & 8.7 & 8.5 & 9.5 \\
\hline & $\max$ & 11.3 & 18.3 & 16.3 & 9.0 & 10.3 & 10.0 & 11.6 \\
\hline & $\sigma$ & 1.3 & 1.4 & 1.3 & 0.9 & 1.0 & 0.8 & 1.8 \\
\hline \multirow[t]{4}{*}{$h s w(\mathrm{~m})$} & $\min$ & 1.0 & 1.0 & 1.1 & 0.9 & 0.9 & 0.6 & 0.3 \\
\hline & $\bar{x}$ & 1.9 & 1.3 & 1.5 & 1.9 & 2.0 & 1.5 & 0.6 \\
\hline & $\max$ & 3.2 & 1.7 & 2.2 & 2.7 & 2.9 & 2.6 & 0.8 \\
\hline & $\sigma$ & 0.8 & 0.1 & 0.2 & 0.6 & 0.6 & 0.5 & 0.2 \\
\hline \multirow[t]{4}{*}{$T s w(s)$} & $\min$ & 4.2 & 6.0 & 5.3 & 3.2 & 3.3 & 11.4 & 3.4 \\
\hline & $\bar{x}$ & 9.6 & 8.7 & 8.2 & 11.1 & 11.9 & 12.1 & 4.9 \\
\hline & $\max$ & 13.5 & 13.0 & 14.2 & 16.5 & 16.8 & 14.4 & 6.8 \\
\hline & $\sigma$ & 4.5 & 2.3 & 2.5 & 5.0 & 4.7 & 0.8 & 1.7 \\
\hline \multirow[t]{4}{*}{$C s w / U_{10 N}$} & $\min$ & 0.6 & 1.1 & 0.8 & 0.5 & 0.5 & 1.9 & 0.4 \\
\hline & $\bar{x}$ & 4.3 & 2.9 & 2.5 & 2.8 & 2.7 & 4.6 & 1.5 \\
\hline & $\max$ & 11.0 & 10.1 & 6.9 & 5.3 & 6.5 & 11.3 & 3.6 \\
\hline & $\sigma$ & 3.5 & 1.7 & 1.1 & 1.2 & 1.4 & 2.5 & 1.2 \\
\hline \multirow{3}{*}{$\begin{array}{l}h s w / L s w \\
\times 10^{3}\end{array}$} & $\bar{x}$ & 20 & 14 & 18 & 18 & 22 & 7 & 19 \\
\hline & med. & 9 & 17 & 18 & 7 & 7 & 7 & 25 \\
\hline & $\sigma$ & 7 & 6 & 6 & 6 & 6 & 5 & 12 \\
\hline
\end{tabular}

Note. The terms med. refer to median values, while $\bar{x}$ and $\sigma$ correspond to mean values and to standard deviations, respectively.

self-motion of the platform could sometimes be confused spectrally with the waves signal. In addition, the double integration of $a c c z$ required for calculating $\eta$ and $T s w$ was sometimes biased at time periods larger than 15-20 s because of their relatively low signal-to-noise ratio. The calculated values of Tsw correspond to the periods where the maximum of the velz spectra occurred. We are aware that these values are not the true Tsw values, but only an estimation of them. We were more confident in the location of the maxima of velz spectra than in the location of the maxima of water elevation spectra, because velz time series were directly acquired on board, while elevation time series were processed through a cumulative integration of the velocities. The daily Tsw values found with this approach range from 4.9 to $12.1 \mathrm{~s}$, as reported in Table 2, which seems in agreement with visual observations. However, in spite of our efforts, bad detection of Tsw still persists with some of the data. They result in unrealistically large values of Tsw that are typically equal or larger than $14 \mathrm{~s}$, which would correspond to wavelengths larger than $300 \mathrm{~m}$ (maximum values of Tsw in Table 2).

Wave age is here defined as the ratio $C s w / U_{10 N}$, where $C s w$ is the phase speed of sea waves at their peak frequency, itself defined as $(\mathrm{g} / 2 \pi) T s w$ for deep water waves and where $U_{10 N}$ is the reference wind speed at an 
altitude $z=10 \mathrm{~m}$, in neutral conditions of stability, calculated with the EC algorithm. The wave age values are essentially larger than unity, as reported in Table 2 , which means that most data indicate swell conditions, that is, waves travelling faster than wind. The overall value of $C s w / U_{10 N}$ averages $2.8 \pm 1.6$.

Sea conditions may lastly be described in terms of wave steepness, a proxy of which is defined here as $h s w /$ $L s w$, where $L s w$ is the wavelength of the most significant waves itself defined as the product CswTsw. As reported in Table 2, the median values of $h s w / L s w$ are larger by a factor 2 or more for S12, A14, and U17 data than for the other experiments. Please note that this is not true for mean values, as we reach here the limits of confidence of our data.

\subsection{Vertical Velocity Correction}

As mentioned in the introductory review, a direct subtraction (DS) method (Pedreros et al., 2003) may not be enough to correct wind data from platform motion at water wave scales and at atmospheric turbulent scales. Although OCARINA is a wave-following platform with small vertical motion, we decided to develop a new and radical correction technique that is presented hereafter.

The proposed technique is based on the rather strong hypothesis that any vertical wind fluctuation that would be spectrally coherent with the vertical platform motion fluctuation should be suppressed. This is written as

$$
\begin{aligned}
& E_{w_{\text {corr }}}(f)=E_{w}(f)-a(f) E_{\text {velz }}(f) \\
& a(f)=\frac{E_{w}(f) \operatorname{conj}\left(E_{\text {velz }}(f)\right)}{\left\|E_{w}(f) \operatorname{conj}(\operatorname{velz}(f))\right\|}
\end{aligned}
$$

where $E_{w}(f)$ and $E_{v e l z}(f)$ are the Fourier transforms of $w$ and velz, respectively, and $E_{w c o r r}(f)$ is the resulting corrected wind component in Fourier space. Time series of corrected vertical wind $w_{\text {corr }}$ are then obtained by applying an inverse Fourier transform to $E_{w \text { corr }}(f)$. In the following, this technique is referred to as spectral coherence (SC) technique. For its application, we used a numerical smoothing over 51 points to the numerator and to the denominator of equation (10), a value adjusted by trial and error. Not only does this technique account for amplitudes as a function of frequency but it also accounts for phase differences between wind and vertical motion at all frequencies, which is much more complete than the standard DS method.

The two above mentioned methods, namely, the DS and SC methods, were applied to the six OCARINA data sets. The power spectra of vertical wind before and after correction averaged over the entire data sets are shown in Figure 3. For F11 data, Figure 3a clearly shows that the DS method efficiently removes the energy from the vertical wind signal at frequencies larger than $\sim 0.3 \mathrm{~Hz}$, which is comparable to what B14 found for the longitudinal wind component. However, at frequencies smaller than $0.3 \mathrm{~Hz}$, our data suggest that the correction does not perform well because the power of the corrected velocity spectrum gradually increases with decreasing frequencies, whereas the raw wind signal decreases (Figure 3a). In contrast, the SC technique removes energy from the wind signal and flattens the motion peak at low frequencies.

The SC method gives comparable results for five other data sets such that the spectral peaks of vertical wind are smoothed when the correction is applied (Figures 3b-3d, 3f, and 3g). As for F11, the subtraction technique performs as well as the SC method for the two data sets S12 and A14, but only at frequencies larger than $0.3 \mathrm{~Hz}$. The case of U17 is indicative on the differences between the two correction methods. The presence of short wind-driven waves during the experiment converts into a well-identified $\left|E_{w}(f)\right|^{2}$ peak centered at 0.3 $\mathrm{Hz}$ in Figure $3 \mathrm{~g}$ (black line). After application of the DS method, the low-frequency part of the peak is not efficiently removed, as found above, whereas the SC method performs better. As there is no immediate reason for the physical processes to significantly differ from either part of the peak, our data suggest in this case that the DS method is not efficient at frequencies smaller than $0.3 \mathrm{~Hz}$.

In the case of B15-2, the supposed motion peak at $\sim 0.5 \mathrm{~Hz}$ is not well corrected by any of the two methods (Figure 3e). On one hand, the DS method rather adds noise at low frequencies, whereas the SC technique does not modify much the original $w$ spectrum because the platform vertical velocity spectrum drops to small values at frequencies larger than $0.2 \mathrm{~Hz}$. In this case, the small impact of this correction technique 

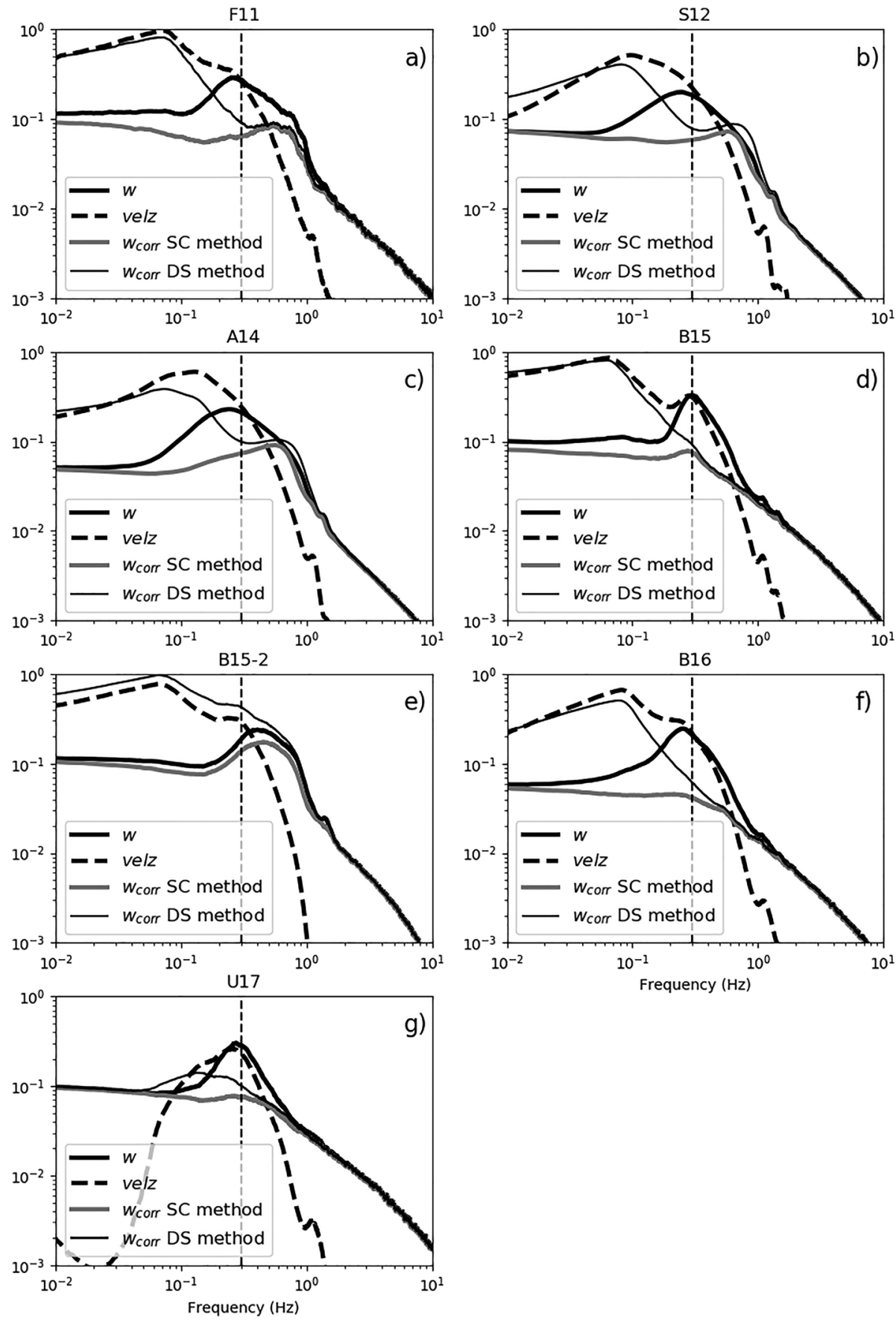

g)

Figure 3. Averaged power spectra (in arbitrary units) of the uncorrected vertical wind component (thick black line), of the vertical velocity of OCARINA (thick dotted lines), of the vertical wind component corrected with the SC method (thick gray line), and of the vertical wind component corrected with the DS method (Pedreros et al., 2003). The vertical dashed line denotes the generally observed limit of efficiency of the subtraction method.

could be attributed either to the lower quality of the accelerometers used or to the particular design shape of the Campbell Irgason anemometer that was used during this experiment.

At this stage, one may not conclude that suppressing the vertical wind peak is necessarily a success. This will be further analyzed with cospectra in section 3.1.3 and from the point of view of the discrepancies between EC, bulk, and ID flux estimates in section 4.1. 


\section{Characteristics of Turbulence}

In this section, we first describe the spectral characteristics of turbulence with wind and temperature power spectra and with wind cospectra. Next, we estimate usual quantities that characterize the turbulent flow, namely, the dissipation rates of energy and the Kolmogorov constant.

\subsection{Spectral Characteristics}

\subsubsection{Wind Inertial Subrange}

Defining the wind inertial subrange is required for calculating ID fluxes. It is done by finding the frequency range of the power spectra of the measured longitudinal wind component $\left|E_{u}(f)\right|^{2}$ that nearly follows a $-5 / 3$ logarithmic slope. According to Figure 4, the wind power spectra normalized as $f^{5 / 3}\left|E_{u}(f)\right|^{2} / U_{r}^{2}$, where $U_{r}$ is the apparent wind value that does not account for platform horizontal velocity, show an undisturbed inertial subrange at 2-7 Hz for OCARINA \#1 and at 1.2-4 Hz for OCARINA \#2. The undisturbed range of the inertial spectra slightly depends on the experiment considered; it is the largest for B15 and the smallest for S12. The wave-induced motion of OCARINA produces peaks in the $u$ spectra at frequencies of about $0.8 \mathrm{~Hz}$. In addition, a flexion mode of the instrument-supporting mast also generates peaks at $9 \mathrm{~Hz}$ for \#1 (also seen in B14) and at $5 \mathrm{~Hz}$ for \#2.

The effect of the most significant waves results in peaks, which are more apparent at $\sim 0.09 \mathrm{~Hz}$ in the normalized spectra of Figures $4 \mathrm{a}-4 \mathrm{c}$. The envelopes of individual spectra reveal only one outlier, happening during B15. Overall, the mean normalized spectra of $u$ represent well the envelopes of individual spectra, apart from U17 that reveals the presence of two types of spectra, which is explained by two situations during the same experiment (upwind of the island of Porquerolles where the conditions were comparable to those in the open ocean and downwind of the Presqu'île de Giens, in very short fetch conditions both for sea waves and for the SABL, as can be seen in the bottom panel of Figure 2). According to the shapes of the wind spectra analyzed in this section, we selected inertial subranges for later ID flux calculations (in section 4.2) within the 2-5- $\mathrm{Hz}$ range, as reported in Table 3 .

\subsubsection{Temperature Inertial Subrange}

The averaged sonic temperature power spectra|$\left|E_{T \text { son }}(f)\right|^{2}$ normalized by the variance of temperature $\sigma_{T \text { son }}{ }^{2}$ and multiplied by $f^{5 / 3}$ are plotted as thick black curves in Figure 5. Please note that a high-pass filter with a normalized cutoff frequency $f z / U$ equal to 0.02 (or 10-20 s) was applied before the calculation of the variances, which dramatically improved the normalization. The normalized spectra presented in Figure 5 almost all present an inflexion point around $1 \mathrm{~Hz}$, which we interpreted as the beginning of the temperature inertial subrange. However, Figure 5 reveals that the averaged spectra do not represent well the envelope of individual spectra. By plotting them as a function of their wind speed range, we observe that the temperature spectral slope is partly dependent on wind intensity, as highlighted by different shades of gray in Figure 5.

Interestingly, stronger winds correspond to steeper slopes, which turn into log exponents smaller than $-5 / 3$ (light gray curves in Figure 5). We find that the inertial subrange is better defined and of larger extent for wind values smaller than $5 \mathrm{~m} / \mathrm{s}$ (dark and medium gray curves), which is more apparent in Figures $5 \mathrm{~b}-5 \mathrm{e}$ and $5 \mathrm{~g}$. Note that there are outlier cases, such as in Figures $5 \mathrm{a}$ and $5 \mathrm{f}$ where the spectral envelopes in medium gray and in dark gray do not comply with this. For the relatively large wind values $(U>7 \mathrm{~m} / \mathrm{s})$, the comparison between Figures $5 \mathrm{~d}$ and $5 \mathrm{e}$ is interesting because they correspond to the same experiment, but with two different instruments (section 2.1).

Although the behavior of the different temperature spectra is comparable at moderate wind speeds (medium gray curves) with a wide and clearly defined subrange that extends from 1 to $10 \mathrm{~Hz}$, we observe discrepancies at wind speeds larger than $7 \mathrm{~m} / \mathrm{s}$. Indeed, the Irgason temperature spectra show smooth peaks at 7-10 Hz, which could also correspond to the inertial temperature subrange, while the Gill R3-50 spectra apparently present aliasing or folding at high frequencies. As a result, we cannot draw firm conclusions on the slope of temperature spectra for the experiments at wind speeds larger than $7 \mathrm{~m} / \mathrm{s}$. Thus, we will use temperature data in the range $1-2 \mathrm{~Hz}$ for calculating ID fluxes for all the experiments. However, for clarity, the 7-10-Hz subrange suspected above for B15-2 data shall be tested in section 4.2.

\subsubsection{Wind Cospectra}

The cospectra between the longitudinal wind component and its vertical counterpart represent the energy distribution of the momentum flux along the mean wind vector and across the entire available range of 

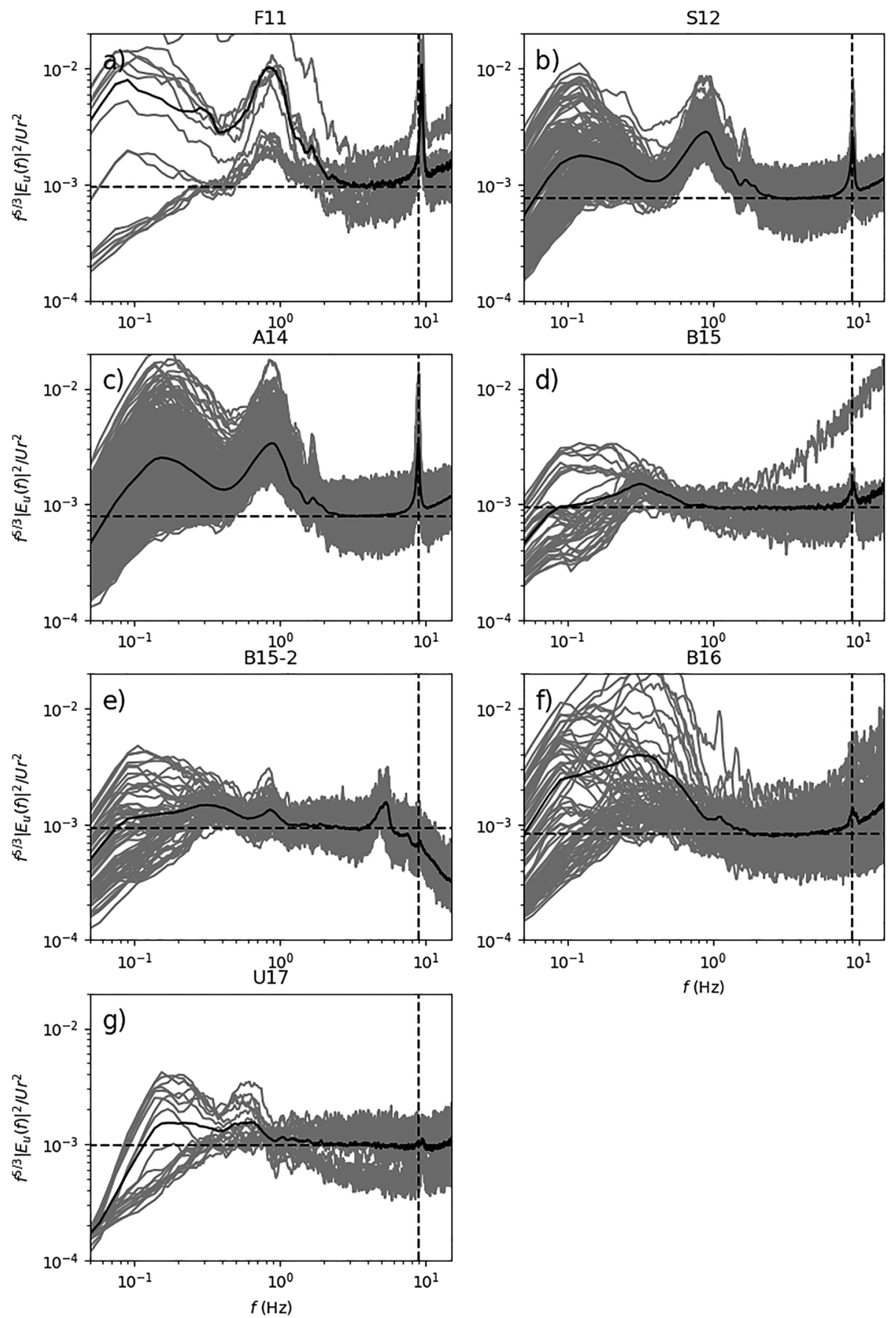

Figure 4. Normalized wind power spectra for each experiment. The black curves show the averaged spectra, the gray overlapping curves represent the envelope of the individual spectra, the vertical dashed lines locate a mast flexion mode that occurs for prototype \#1 at $9.1 \mathrm{~Hz}$, and the horizontal dashed lines locate the mean values of each spectrum at $4 \mathrm{~Hz}$.

frequencies. Kaimal et al. (1972) proposed a model of normalized cospectrum based on data collected over land that is used here as a reference cospectrum.

Calculated cospectra look rather different depending on the normalization used, on the calculation method used, and on the frequency chosen on the $x$ axis. It may be either the absolute frequency $f$ in $\mathrm{Hz}$, a normalized frequency $f z / U$ (which is usual), or a modified frequency scale $f / f_{0}$ where $f_{0}$ is defined in Kaimal et al. (1972). Here, we present cospectra normalized by their integral value and multiplied by $f$. Therefore, the cospectra 
Table 3

Frequency Range Selection for the Wind Inertial Subrange

\begin{tabular}{lccccccc}
\hline Experiment & F11 & S12 & A14 & B15 & B15-2 & B16 & U17 \\
\hline f_min (Hz) & 3 & 3 & 3 & 2 & 2 & 2 & 3 \\
f_max (Hz) & 4 & 5 & 5 & 4 & 3 & 3 & 5 \\
\hline
\end{tabular}

Note. f_min and f_max are the minimum and maximum selected frequencies, respectively. are not normalized as usually done by the square of a friction velocity, which could be affected by various biases depending on the motion correction applied to the vertical wind component. The normalized cospectra may be directly compared to the reference cospectra. The first step in the calculation consists in computing a normalized cospectrum and its associated Kaimal reference cospectrum for each time segment of an experiment. Next, both cospectra are interpolated on a large linear range of 35,000 normalized frequency values $f z / U$ from $10^{-3}$ to 15 , encompassing all the accessible frequency values. In a next step, the resulting interpolated cospectra are independently averaged over all the available time segments. Last, an arbitrary 51-point-long median filter is applied to each averaged data-based cospectrum to produce a smoother thus easier to interpret envelope of points.

Various types of cospectra were computed in this way, namely, (1) with noncorrected vertical motion, (2) with the DS correction, and (3) with the SC correction, following section 2.4 tests. Hereafter, they are referred to as noncorrected, DS, and SC cospectra, respectively.

In addition, for experiments where enough cospectra were available to produce reliable averages ( $\mathrm{S} 12$ and A14), we also performed calculations as a function of stability range. For S12, unstable conditions are defined as $\left|\frac{z}{L}\right|<-0.1$, which occurs for 40 cospectra. We also analyzed data for the neutral-unstable $\frac{z}{L}$ range that is common to S12 and A14 data $\left(-0.01>\frac{z}{L}>-0.05\right)$, which corresponds to 152 and 155 cospectra, respectively. Note that we do not present the analysis of the neutral-stable cases of A14 (section 2.2), because the corresponding cospectra have an inconsistent behavior, which are very sensitive to the $\frac{z}{L}$ thresholds selected (not shown).

As shown in Figure 6, the integral value of the SC cospectrum may considerably depart from the integral of the reference cospectrum, down to $-100 \%$ of difference, as shown in Figures $6 \mathrm{a}$ and $6 \mathrm{~g}$. Nevertheless, the SC cospectra present a bell-like shape for all experiments but F11, the data of which are noisy and sometimes negative, which is not encouraging, contrasts with section 2.4 results, and justifies that more data were required to confirm the results of $\mathrm{B} 14$. For the other experiments, the SC cospectra give consistent thus more encouraging results. In contrast, the application of the DS correction results in decreased and sometimes negative values of the cospectrum at normalized frequencies smaller than 0.05-1 for S12, A14, B15, B15-2, and B16 data (Figures 6b-6f), which is consistent with section 2.4 findings, whereas at higher frequencies, the DS cospectra have large suspect values compared to the noncorrected cospectra in Figures $6 \mathrm{~b}, 6 \mathrm{c}, 6 \mathrm{e}$, and $6 f$.

Interestingly, the application of the SC method to S12 and A14 experiments for which we have most of our data (black curves in Figures $6 \mathrm{~b}$ and $6 \mathrm{c}$ ) only slightly decreases the values at the frequency of the maximum peak of the cospectrum compared to noncorrected data (in orange). This is most obvious for S12 data (Figure 6b), for which no correction would apparently be required for calculating the momentum flux.

As shown in Figures $6 \mathrm{~b}$ and $6 \mathrm{c}$, for all the correction methods used, the maximum frequency peak of the S12 and A14 cospectra is shifted toward larger normalized frequencies compared to the model of Kaimal et al. (1972), by large factors, namely, 2.75 and 4.5, respectively.

As shown in Figure 6b, the frequency of the maximum peak of the SC cospectrum is shifted toward larger frequencies in unstable conditions compared to near-neutral conditions (the red curve in Figure $6 \mathrm{~b}$ is located to the right of the green curve), and the amplitude of the cospectra is larger in unstable conditions than in near-neutral conditions, which was also documented in Cambra (2015).

For the neutral-unstable $z / L$ range shared by S12 and A14 data, that is, $-0.01>\frac{z}{L}>-0.05$, the S12 and A14 cospectra peak at a normalized frequency of $0.2-0.22$, which is close thus encouraging (green curves in Figures $6 \mathrm{~b}$ and $6 \mathrm{c})$.

The frequency shifts of the S12 and A14 SC cospectra with respect to the reference cospectra have to be explained by another factor than stability, because they also occur in neutral conditions (green curves in Figures $6 \mathrm{~b}$ and $6 \mathrm{c}$ ). This could be related to the wave-following nature of the OCARINA platform, whose 

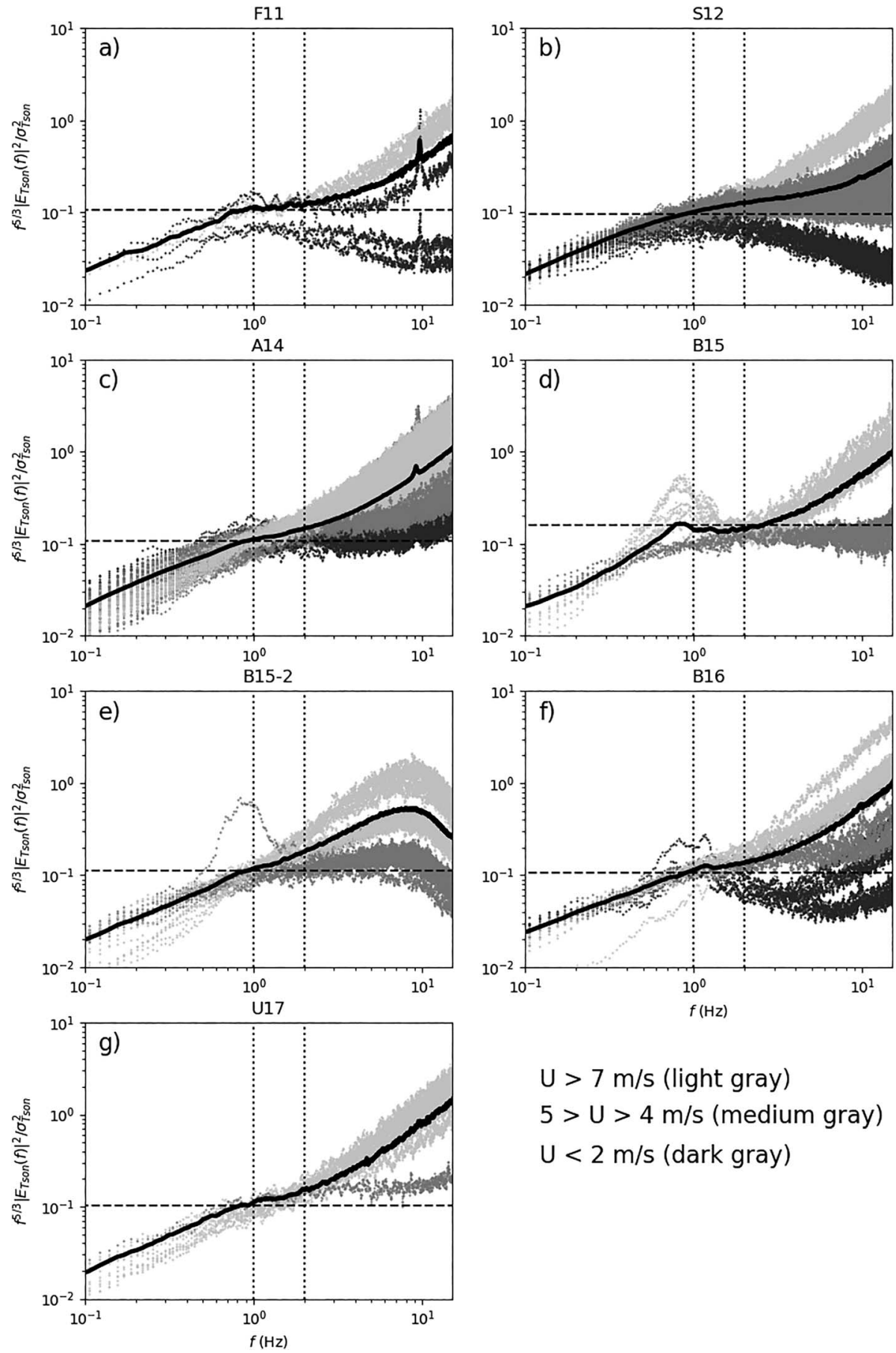

$$
\begin{aligned}
& \mathrm{U}>7 \mathrm{~m} / \mathrm{s} \text { (light gray) } \\
& 5>\mathrm{U}>4 \mathrm{~m} / \mathrm{s} \text { (medium gray) } \\
& \mathrm{U}<2 \mathrm{~m} / \mathrm{s} \text { (dark gray) }
\end{aligned}
$$

Figure 5. Normalized sonic temperature spectra for each experiment. The black curves show the averaged spectra, and the horizontal dashed lines locate the mean value of each spectrum at $1 \mathrm{~Hz}$. The vertical dotted lines encompass the inertial frequency subrange selected for temperature.

drifting velocity with respect to the sea surface is not measured (section 2.1). Note also that the normalized frequency used by Kaimal et al. (1972) may not fully apply over waves. Indeed, their presence overall focuses energy and momentum transfers across the interface on particular scales. This may be quantified in various ways, such as in terms of effective to peak phase speed ratio, as suggested by CifuentesLorenzen et al. (2018). Although finding an appropriate frequency scale was not the focus in this paper, the larger median wave steepness estimates found for S12 and A14 data compared to the other swell- 

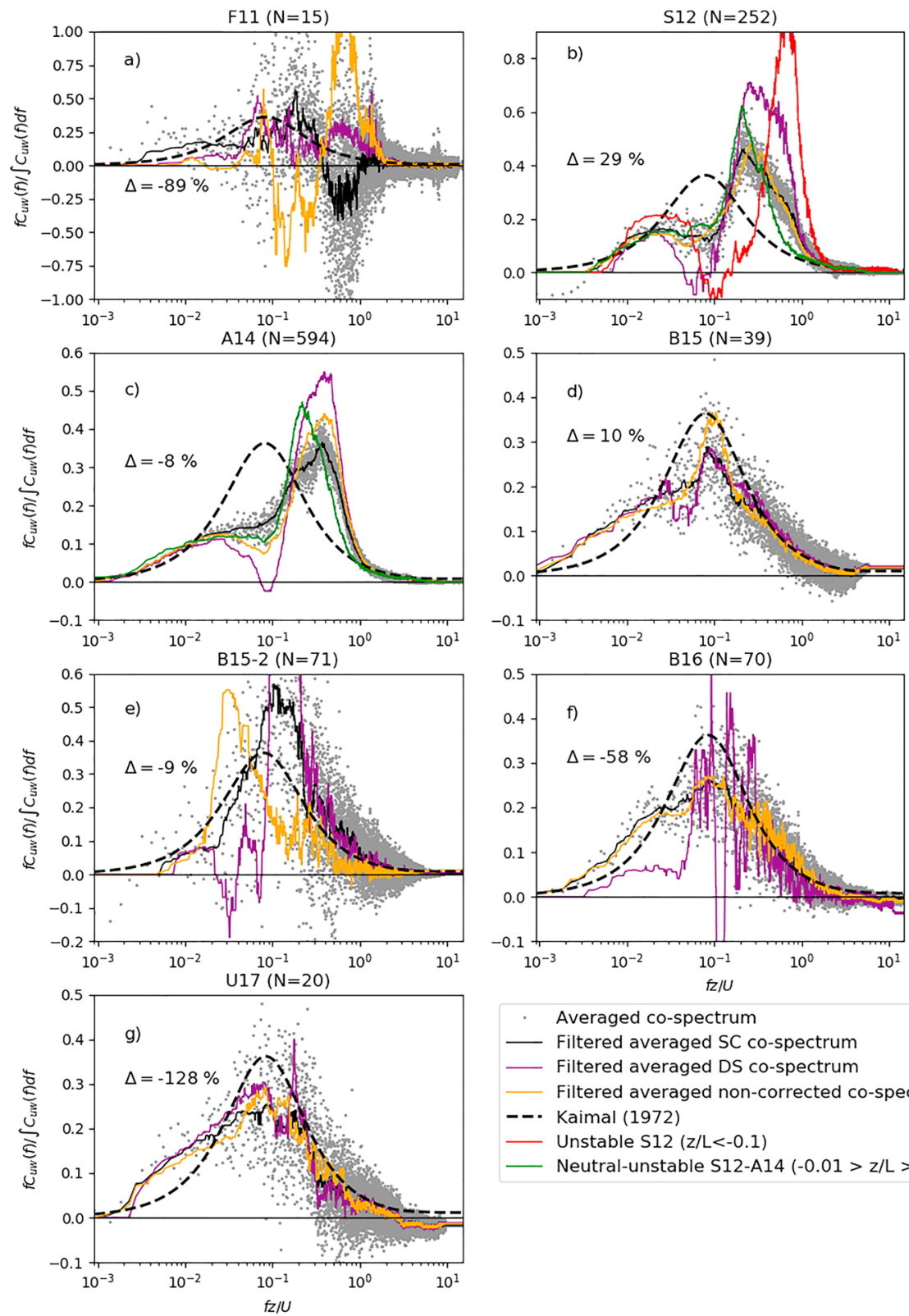

Averaged co-spectrum

- Filtered averaged SC co-spectrum

- Filtered averaged DS co-spectrum

_ Filtered averaged non-corrected co-spectrum

--. Kaimal (1972)

- Unstable S12 (z/L<-0.1)

- Neutral-unstable S12-A14 (-0.01 > z/L > -0.05)

Figure 6. Normalized cospectra for the longitudinal wind component (gray dots). The values of $N$ in parentheses indicate the number of SC cospectra available for averaging. The dashed curve is the Kaimal et al. (1972) model. The thin black lines correspond to the application of a low-frequency median filter to the gray dots, through all frequencies. The symbol $\Delta$ denotes the percentage of difference between the integral values of the averaged SC cospectra with respect to the integral values of the Kaimal et al. (1972) model. Cospectra for the noncorrected and the DS-corrected vertical velocity components are represented in orange and purple, respectively. For S12 and A14 experiments, cospectra are also represented as a function of stability ranges, that is, unstable (red) and neutral-unstable (green).

dominated experiments (section 2.3) also support that a wave-related frequency scale would be more appropriate than $f z / U$ for modeling the wind cospectra.

\subsection{Integral Characteristics}

\subsubsection{Dissipation Rates}

The calculation of the dissipation rate of turbulence is required for estimating ID $u_{*}$, as already mentioned in section 1.2. However, in addition to inferring the dissipation rate of turbulence from the wind power spectrum (equation (5)), it can also be calculated with the time-dependent third-order structure 
function $S_{3}(\mathrm{dt})$, as proposed by Fairall et al. (2003). This- under the assumption of frozen turbulence (e.g., B09)—may be written as

$$
S_{3}(\mathrm{dt})=\overline{(u(\mathrm{t}+\mathrm{dt})-u(\mathrm{t}))^{3}}=-\frac{4}{5} \varepsilon_{3} U_{r} \mathrm{dt}
$$

where the variable $t$ is the time, $\mathrm{d} t$ is a time increment, the maximum extent of which is from the duration of a half-time segment (550 s) to the inverse of the Nyquist frequency $(1 / 16 \mathrm{~Hz})$, and $\varepsilon_{3}$ is the dissipation rate of turbulence. Note that the equivalent of equations (5) and (11) for temperature are

$$
\begin{gathered}
\varepsilon_{\theta}=\varepsilon^{\frac{1}{3}}\left(\frac{2 \pi}{U_{r}}\right)^{\frac{2}{3}}\left(\frac{E_{\theta}(f)}{c_{\theta}}\right) f^{\frac{5}{3}} \\
S_{3 \theta}(\mathrm{dt})=\overline{(u(\mathrm{t}+\mathrm{dt})-u(\mathrm{t}))(\theta(\mathrm{t}+\mathrm{dt})-\theta(\mathrm{t}))^{2}}=-\frac{4}{3} \varepsilon_{3_{\theta}} U_{r} \mathrm{dt}
\end{gathered}
$$

where $E_{\theta}(f)$ is the temperature power spectrum, $\varepsilon_{\theta}$ is now called the half-variance rate of destruction of temperature, $c_{\theta}$ is the Corrsin constant $(0.8)$, and $S_{3 \theta}(\mathrm{dt})$ is the time-dependent wind-temperature third-order structure function.

Hereafter, we compare the calculated values of $\varepsilon$ (resp. $\varepsilon_{\theta}$ ) to the values of $\varepsilon_{3}$ (resp. $\varepsilon_{3_{\theta}}$ ). This gives a way to assess our choices in the application of the ID method, as the comparisons depend on the choice of the frequency bands of the inertial subrange made in section 3.1.

Here, dissipation rates for the longitudinal wind component $\varepsilon$ and $\varepsilon_{3}$ are calculated either as mean values over the inertial subrange selected in section 3.1 or as maximum values over the whole frequency range, which includes inappropriate values in the turbulent production range, although this has no impact on the results (not shown). They are plotted versus the dissipation calculated from the spectra in the inertial subrange in Figures 7a and 7c. The best agreement is found with mean values (Figure 7a), specifically for the bias that is lowered by $48 \%$ compared to when choosing maximum values (Figure $7 \mathrm{c}$ ). The correlation coefficient found between $\varepsilon$ and $\varepsilon_{3}$ is 0.94 , which is good, and the rms deviation of the difference between $\varepsilon$ and $\varepsilon_{S 3}$ is $4 \times 10^{-3} \mathrm{~m}^{2} / \mathrm{s}^{3}$. This is comparable thus not better than what was found earlier using an instrumented mast on a large ship, that is, 0.85 and $4 \times 10^{-3} \mathrm{~m}^{2} / \mathrm{s}^{3}$, respectively (B09). Hence, this comparison between $\varepsilon$ and $\varepsilon_{3}$ confirms the consistency of the inertial subrange chosen for the ID method.

The comparisons between $\varepsilon_{\theta}$ and $\varepsilon_{3_{\theta}}$ values are presented in Figures $7 \mathrm{~b}$ and $7 \mathrm{~d}$. In opposite to the case for the dissipation rates, the best agreement is clearly found with maximum values of the structure function, which strongly improves the slope of the linear fit, by $57 \%$. The correlation coefficient found between $\varepsilon_{\theta}$ and $\varepsilon_{3_{\theta}}$ is 0.96 , which is good, and the rms difference found is $0.1 \mathrm{~K}^{2} / \mathrm{s}$, which is acceptable though it is strongly constrained by distribution of the data that is denser at low values. In opposite to the case of dissipation rate TKE, Figure 7d indicates that the inertial subrange limits selected in section 3.1 for compliance of the temperature power spectra with the MO theory do not compare so well to the inertial subrange viewed from third-order structure functions.

3.2.2. Kolmogorov Constant

The Kolmogorov constant, c, may be expressed as (e.g., B09)

$$
c=0.55 \varepsilon_{3}^{\frac{2}{3}} \varepsilon^{-\frac{2}{3}}
$$

With our data, the values of $c$ calculated with equation (14) range from 0.36 to 0.63 and are variable from one experiment to another, as reported in Table 4. The values reported here result from a selection of $c$ values in the range $[0 ; 1.5]$. The overall mean value found for is $0.48 \pm 0.16$, which is $13 \%$ smaller than the standard 0.55 value. The values depend on the frequency band selection, with values varying from 0.3 to 1 , according to the subrange limits selected. Specifically, we found that they slightly increase with frequency (not shown).

\section{Turbulent Fluxes}

Here, we first compare turbulent fluxes that were successively calculated with the ID, EC, and bulk methods. In agreement with the results of section 2.4, we produced two sets of EC estimates that were respectively 

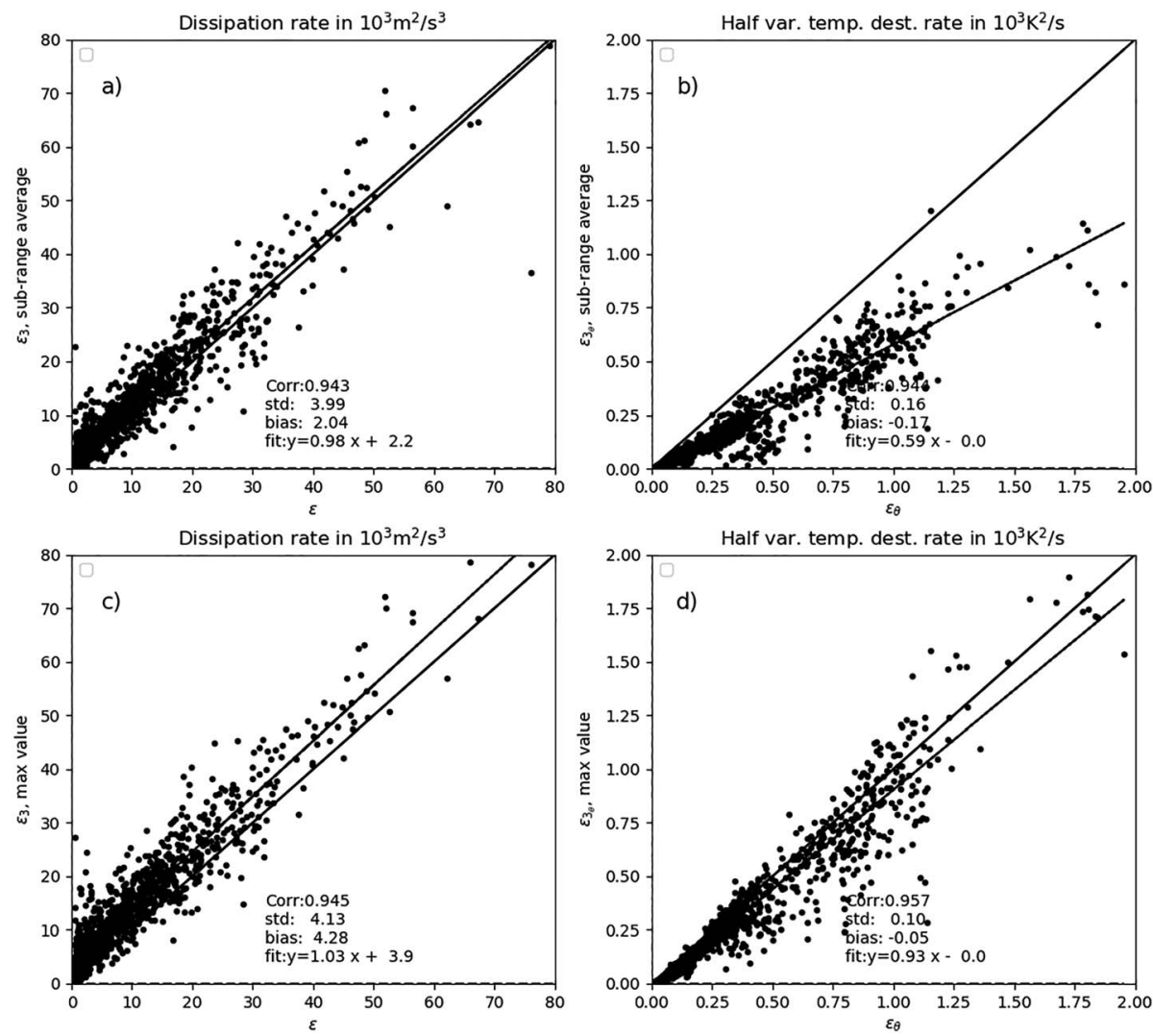

Figure 7. Dissipation rates of turbulence (panels a and c) and half-variance destruction rates of sonic temperature (panels $\mathrm{b}$ and $\mathrm{d}$ ). In panels $\mathrm{a}$ and $\mathrm{b}$, the mean value of the third-order structure function over the inertial subrange is calculated, while in panels $\mathrm{c}$ and $\mathrm{d}$ the maximum value of the third-order structure function is calculated.

based on uncorrected time series of $u, v$, and $w$ (section 2) and on spectrally corrected time series of $w$ and uncorrected $u$ and $v$ values. They are all estimated over 1,100-s time segments, a value that was already successfully used in B09 and B14. We produced two sets of ID flux estimates, respectively, with dissipation rates from power spectra and from third-order structure functions. In the latter case, the calculated dissipation rates (referred to as ID S3 hereafter) were taken equal to the mean value of the dissipation rate spectra in the selected inertial subrange, while the half-variance destruction rates of sonic temperature were taken as the maximum values of the destruction rate spectra over all frequencies, in agreement with the results of section 3.2.1. No imbalance term was taken into account in the ID calculation. In the ID method, the first guess values of $u_{*}$ chosen to solve iteratively equation (6) are the EC estimates, mostly for sign determination. In the bulk method, we used the constant Large and Pond (1981) parameterization of the drag coefficient, because as a first test it is an acknowledged reference at least for winds of medium intensity.

\subsection{Friction Velocity Comparison and Parameter Adjustment in the EC and ID Methods}

When all experiments are considered together, the comparisons between EC, ID, and bulk $u_{*}$ estimates have correlation coefficients larger than 0.9 whether the motion correction is applied or not, which is rather satisfactory (Table 5). The rms differences obtained are in the range $2.4-2.9 \mathrm{~cm} / \mathrm{s}$, which is acceptable, since

Table 4

Statistics of the Kolmogorov Constant (c) Estimates for Each Experiment

\begin{tabular}{lcrrrrrr}
\hline Experiment & F11 & S12 & A14 & B15 & B15-2 & B16 & U17 \\
\hline$c$ & $\bar{x}=0.36$ & $\bar{x}=0.48$ & $\bar{x}=0.45$ & $\bar{x}=0.63$ & $\bar{x}=0.50$ & $\bar{x}=0.55$ & $\bar{x}=0.52$ \\
& $\sigma=0.16$ & $\sigma=0.13$ & $\sigma=0.17$ & $\sigma=0.18$ & $\sigma=0.10$ & $\sigma=0.22$ & $\sigma=0.12$ \\
\hline
\end{tabular}


Table 5

Comparison Between ID, Bulk, and EC Friction Velocity Estimates for the Whole Set of Data

\begin{tabular}{lcccc}
\hline \multicolumn{1}{l}{ All experiments } & & & \\
\hline$u_{*}$ compared & Correlation coefficient & Rms deviation $(\mathrm{cm} / \mathrm{s})$ & Mean deviation $(\mathrm{cm} / \mathrm{s})$ & Slope of linear fit \\
\hline bulk-EC & 0.92 & 2.8 & -0.76 & 0.86 \\
bulk-EC_corr & 0.91 & 2.9 & $0.0(+0.8)$ & 0.92 \\
ID-EC & 0.95 & 2.4 & -4.4 & 0.81 \\
ID-EC_corr & 0.93 & 2.4 & $-3.6(+0.8)$ & 0.87 \\
\hline
\end{tabular}

Note. The values in parentheses indicate the deviation to uncorrected data. The labels EC_corr denote $u *$ estimates corrected with the SC method (see text).

it is on the order of $15 \%$ of the mean $u_{*}$ value. The slopes of linear fits range from 0.81 to 0.92 . The best results in terms of slopes and mean deviation are found when the motion correction in the EC method is applied (Table 5). In contrast, the rms differences are unchanged (ID - EC) or increased by 7\% (bulk EC). An analysis of the comparisons for the different experiments confirms that the changes in the bias are indeed the main outcome from the application of the motion correction. Indeed, the bias is decreased in 11 cases out of 14, as reported in Table 6 column 4. Larger biases are found only in the comparisons between bulk and EC for S12, B15, and B16 data. For the other cases, a smaller bias is gained but the rms differences are smaller or similar only in 4 cases out of these 11 cases. These results suggest that the application of the motion correction to individual data may not be the best approach with our data. We consider that they rather give the magnitude and the sign of a bias to be applied to EC data for each experiment, which is the correction method that is finally adopted hereafter. Technically, we apply a simple mean correction to EC $u *$ of $-1.2 \mathrm{~cm} / \mathrm{s}$ to all data sets but the B15-2 data, for which the correction is set to $-2.9 \mathrm{~cm} / \mathrm{s}$. These two values correspond to the mean values of the bias differences found with and without motion correction (average of the values in parentheses in Table 6). It may be argued here that the application of a simple mean correction is counterproductive with respect to our efforts to set up the SC method in the earlier sections. However, the overall efficiency of this approach shall be further shown in section 5.2 .

Figure 8 shows the friction velocities corrected with the above mentioned mean biases. The resulting values shown in Figures 8a and 8b compare slightly better to bulk and ID estimates of $u_{*}$ in rms deviation and correlation coefficient than in Table 5. Note, however, that the slopes of first degree fits are slightly degraded. There is a positive mean deviation of bulk $u *$ values compared to EC, by $0.6 \mathrm{~cm} / \mathrm{s}$, which should now be attributed to the bulk parameterization chosen, in the hypothesis that the motion correction applied to the EC $u_{*}$ estimates is valuable, which we shall consider in the following.

Figure 8a first reveals that the bulk parameterization chosen is rather appropriate because the correlation coefficient, rms deviation, and slope of linear fit are good, that is, $0.93,2.6 \mathrm{~cm} / \mathrm{s}$, and 0.9 , respectively. In contrast, the parameterization chosen overestimates the mean value of the fluxes by $0.6 \mathrm{~cm} / \mathrm{s}$, which will be further analyzed in section 4.3. Second, EC $u_{*}$ estimates for B15-2 data marked in gray in Figure 8 are underestimated with respect to other data in spite of the correction applied. Third, the ID estimates of $u_{*}$ are in average underestimated compared to EC estimates, by $-3 \mathrm{~cm} / \mathrm{s}$ (Figure 8b), which is in appearance consistent with the small average value of $c$ found in section 3.2.2. Alternatively, an imbalance term could be accounted for in equation (6).

The rms deviation between ID and bulk $u *$ values is $1.4 \mathrm{~cm} / \mathrm{s}$, which is small (Figure 8c), as opposed to the rms deviation found between ID S3 and bulk estimates of $u_{*}$ (Figure 8d), which is twice larger. One might argue that ID S3 fluxes shall not be considered further. However, they show that the mean deviations and slopes are comparable in Figures $8 \mathrm{c}$ and $8 \mathrm{~d}$. This indicates that the modification of $c$ constant does not fully explain why ID or ID S3 $u_{*}$ values are underestimated compared to bulk or EC values of $u_{*}$. Indeed, we have shown that dissipation rates from third-order structure functions were larger than those obtained from power spectra by $2 \times 10^{-3} \mathrm{~m}^{2} / \mathrm{s}^{3}$ (positive bias in Figure 7a). Thus, the bias is indeed decreased if ID S3 is used instead of ID, but the ID S3 are anyway underestimated by $2.2 \mathrm{~cm} / \mathrm{s}$ (Figure 8d), which is significant. In addition, the slope of linear fit is 0.77 in Figure $8 \mathrm{~d}$, which is noticeably smaller than unity. Therefore, it 
Table 6

Comparison Between ID, Bulk, and EC Friction Velocity Estimates for Each Experiment

\begin{tabular}{|c|c|c|c|c|}
\hline$u *$ compared & Correlation coefficient & Rms deviation $(\mathrm{cm} / \mathrm{s})$ & Mean deviation $(\mathrm{cm} / \mathrm{s})$ & Slope of linear fit \\
\hline \multicolumn{5}{|l|}{ F11 data } \\
\hline bulk-EC & 0.95 & 3.5 & -5.1 & 0.95 \\
\hline bulk-EC_corr & 0.95 & $3.5(+0.0)$ & $-3.1(+2.0)$ & 0.92 \\
\hline ID-EC & 0.97 & 2.8 & -7.2 & 0.84 \\
\hline ID-EC_corr & 0.98 & $2.9(+\mathbf{0 . 1})$ & $-5.3(+1.9)$ & 0.83 \\
\hline \multicolumn{5}{|l|}{ S12 data } \\
\hline bulk-EC & 0.96 & 1.6 & 0.0 & 0.95 \\
\hline bulk-EC_corr & 0.89 & $2.6(+1.0)$ & $1.4(+1.4)$ & 0.95 \\
\hline ID-EC & 0.96 & 1.7 & -3.7 & 0.83 \\
\hline ID-EC_corr & 0.87 & $2.5(+0.8)$ & $-2.2(+1.5)$ & 0.82 \\
\hline \multicolumn{5}{|l|}{ A14 data } \\
\hline bulk-EC & 0.96 & 1.8 & -0.3 & 0.99 \\
\hline bulk-EC_corr & 0.94 & $2.2(+0.4)$ & $-0.2(+0.1)$ & 1.08 \\
\hline ID-EC & 0.98 & 1.4 & -4.1 & 0.91 \\
\hline ID-EC_corr & 0.97 & $1.5(+0.1)$ & $-4.0(+0.1)$ & 1.00 \\
\hline \multicolumn{5}{|l|}{ B15 data } \\
\hline bulk-EC & 0.94 & 2.4 & 0.8 & 0.68 \\
\hline bulk-EC_corr & 0.96 & $1.6(-0.8)$ & $1.8(+\mathbf{1 . 0})$ & 0.78 \\
\hline ID-EC & 0.89 & 2.8 & -4.0 & 0.68 \\
\hline ID-EC_corr & 0.93 & $2.0(-0.8)$ & $-3.0(+1.0)$ & 0.80 \\
\hline \multicolumn{5}{|l|}{ B15-2 data } \\
\hline bulk-EC & 0.81 & 3.4 & -7.5 & 1.00 \\
\hline bulk-EC_corr & 0.73 & $4.3(+\mathbf{0 . 9})$ & $-4.6(+2.9)$ & 0.75 \\
\hline ID-EC & 0.86 & 3.0 & -9.8 & 1.02 \\
\hline ID-EC_corr & 0.84 & $3.3(+0.3)$ & $-6.8(+3.0)$ & 0.82 \\
\hline \multicolumn{5}{|l|}{ B16 data } \\
\hline bulk-EC & 0.95 & 2.6 & 0.1 & 0.96 \\
\hline bulk-EC_corr & 0.95 & $2.5(-0.1)$ & $1.2(+\mathbf{1 . 1})$ & 0.98 \\
\hline ID-EC & 0.94 & 2.9 & -3.1 & 0.85 \\
\hline ID-EC_corr & 0.95 & $2.6(-0.3)$ & $-2.1(+1.0)$ & 0.88 \\
\hline \multicolumn{5}{|l|}{ U17 data } \\
\hline bulk-EC & 1.00 & 2.3 & -1.6 & 0.85 \\
\hline bulk-EC_corr & 1.00 & $1.5(-0.8)$ & $0.1(+1.5)$ & 0.91 \\
\hline ID-EC & 1.00 & 3.4 & -6.4 & 0.76 \\
\hline ID-EC_corr & 1.00 & $2.6(0.0)$ & $-4.3(+2.1)$ & 0.81 \\
\hline
\end{tabular}

Note. Values in parentheses indicate the difference found for the considered statistical parameter when the correction is applied.

seems reasonable to conclude that an imbalance term is-or at least is also-required for correcting ID or ID S $3 u_{*}$ estimates with respect to EC $u_{*}$ estimates. To verify this, the imbalance term was estimated according to equation (6), rewritten as

$$
\phi_{\mathrm{IMB}}=\phi\left(\frac{z}{L}\right)-\frac{z}{L}-\kappa z u_{*}{ }^{-3} \varepsilon_{U}
$$

in which $u *$ values here are output from the EC method. The values found for $\phi_{\mathrm{IMB}}$ average to $0.4 \pm 0.15$. Note that $5 \%$ of the absolute values larger than unity were considered as outliers with respect to the distribution of values, and removed. The average value found does not clearly depend either on the experiment considered or on stability (not shown). This is new compared to the existing parameterizations that are a function of $\frac{z}{L}$ and that do not have a constant part. For example, we tested the $-0.46 \frac{z}{L}$ imbalance term proposed by B09 but it produced insignificant changes on our data (not shown).

Applying a 0.4 constant imbalance term to the ID method was then tested and proved more successful. Indeed, the mean deviation between ID and EC $u_{*}$ estimates is almost canceled $(0.08 \mathrm{~cm} / \mathrm{s})$ and the slope of linear fit is equal to 0.98 , while the rms deviation does not change, that is, $2.19 \mathrm{~cm} / \mathrm{s}$. This shall be further discussed in section 5.1. We also tested the application of a Kolmogorov constant estimated from the comparison between dissipation rates (0.48; see section 3.2.2), but we found that it is not as efficient as the use 

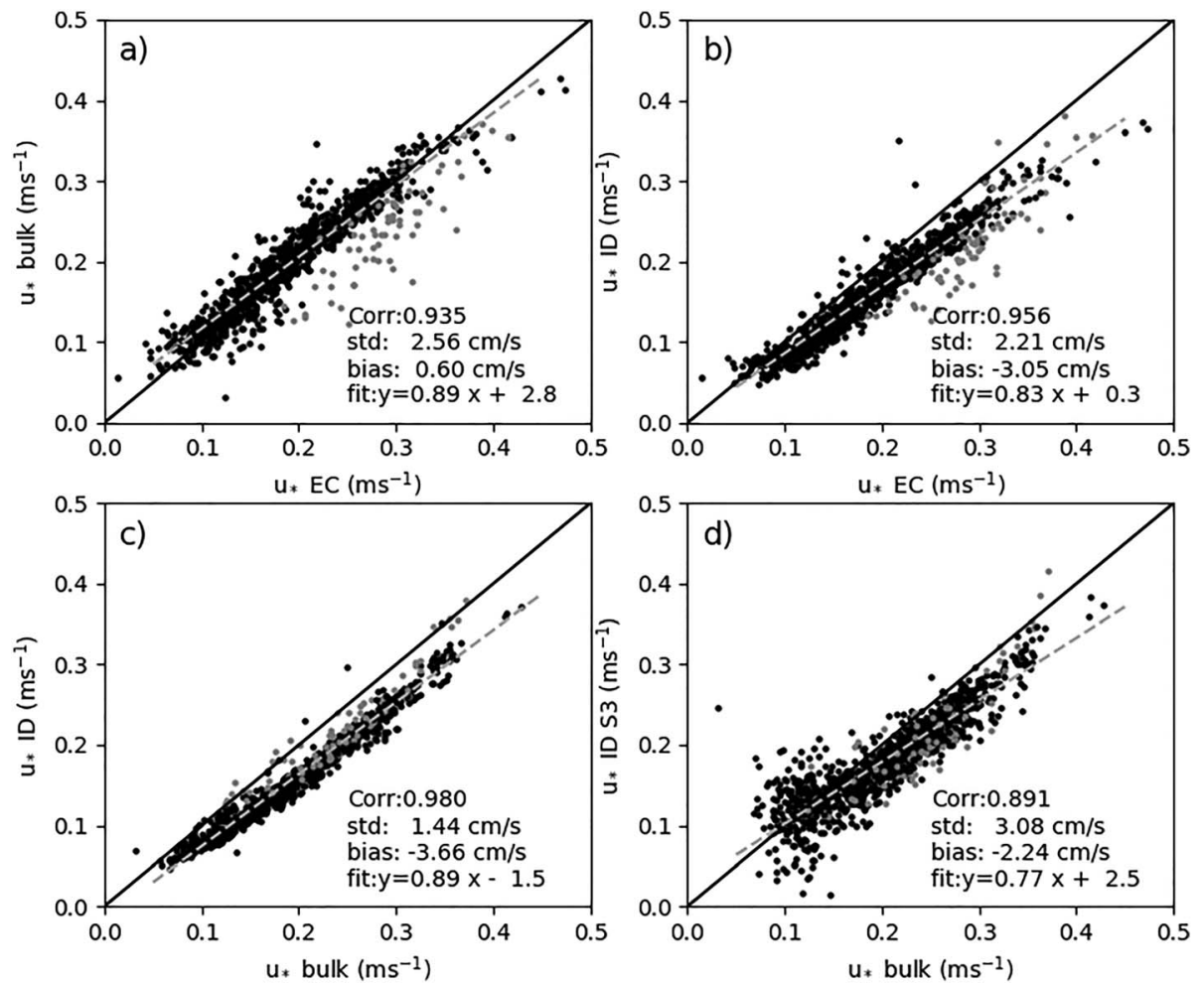

Figure 8. Comparison between friction velocity calculations. Averaged motion correction was applied to EC data. The dots in gray denote B15-2 data. In panel d, the bulk $u^{*}$ estimates are compared to the ID estimates obtained with third-order structure functions (ID S3; see text).

of an imbalance term (not shown). Specifically, the bias is not totally removed between ID and EC $u_{*}$ estimates $(-1.85 \mathrm{~cm} / \mathrm{s})$ and the slope of linear fit is too small compared to estimates corrected with the 0.4 imbalance term $($ slope $=0.89)$.

\subsection{Buoyancy Flux Comparison}

Estimates of $H_{S V}$ obtained with the ID, ID S3, EC, and bulk methods are compared to each other in Figure 9. The rms differences found between the different types of $H_{S V}$ estimates range from 2.8 to 3.1 $\mathrm{W} / \mathrm{m}^{2}$, which denotes a surprisingly good agreement between data, specifically for the ID and ID S3 methods in spite of the possible deviation of the temperature power spectra from the $-5 / 3$ slope at large wind speeds (Figure 5). Noticeably, the slopes of linear fits to data have small deviations to unity in Figures 9a and $9 \mathrm{~b}$, that is, 0.98 to 1.13 . Note that the 0.4 imbalance term found in the last section was applied to the ID calculation presented above, which has an impact on ID $H_{S V}$ estimates through the modification of $u_{*}$ thus of $\frac{z}{L}$ during the iterative calculation (e.g., B09). If the imbalance term is not accounted for, then the slope between $H_{S V}$ from ID and EC decreases to 0.91 (not shown). We conclude from the above results that the proposed imbalance term is sufficient to correct ID $H_{S V}$ values with respect to EC estimates. In contrast, the slope of linear fit is only $0.79-0.84$ between ID or IDS3 and bulk estimates as shown in Figures 9c and 9d.

In section 3.1.2, we questioned the location of the temperature inertial subrange for B15-2 data, because the corresponding temperature power spectrum presented a $-5 / 3$ slope in the range $7-10 \mathrm{~Hz}$ (Figure 5e), while a $1-2-\mathrm{Hz}$ band was eventually selected for all the experiments. In response, we performed additional calculations of ID $H_{S V}$ values using the 7-10-Hz band for B15-2 data. We found disappointing results; namely, the rms differences between the ID $H_{S V}$ estimates and the bulk or the EC $H_{S V}$ estimates were larger than 10 $\mathrm{W} / \mathrm{m}^{2}$, and the slope of linear fit was 1.4-1.8 (not shown), which confirms that the $7-10 \mathrm{~Hz}$ is not an appropriate choice for the temperature inertial subrange. 

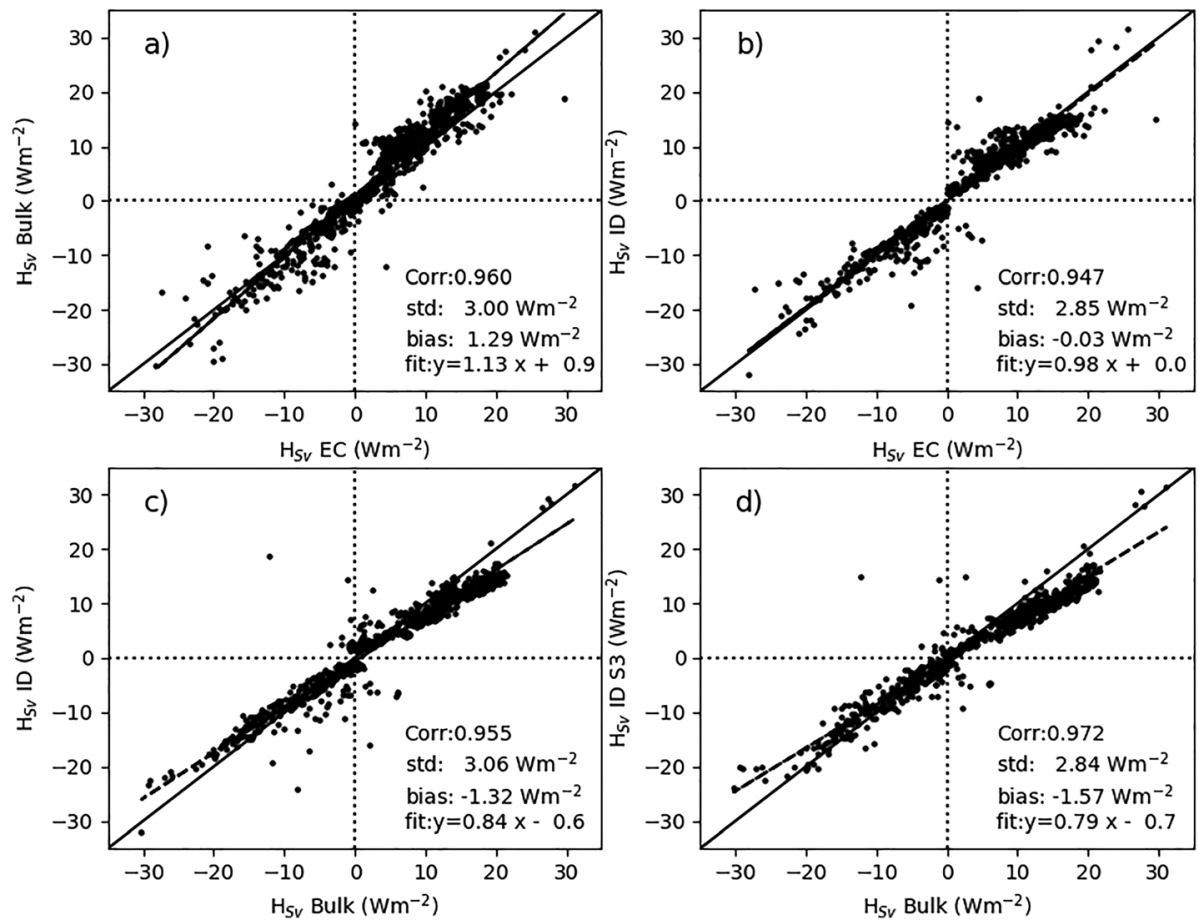

Figure 9. Comparisons for the buoyancy flux.

\subsection{Exchange Coefficients}

\subsubsection{Drag Coefficient}

The equivalent $10-\mathrm{m}$ neutral wind $U_{N 10}$ and drag coefficient $C_{D_{N 10}}$ are calculated with wind module data at the height of measurement $z$, and with EC estimates of $u_{*}$ and $\frac{z}{L}$. In a first step, $z_{0}$ is calculated as

$$
z_{0}=z \exp \left(-\kappa \frac{U}{u_{*}}-\psi\left(\frac{z}{L}\right)\right)
$$

In a second step, $C_{D_{N 10}}$ and $U_{N 10}$ are estimated considering that friction velocity and roughness length are unchanged

$$
\begin{aligned}
C_{D_{N 10}} & =\kappa^{2} / \ln \left(10 / z_{0}\right)^{2} \\
U_{10 N} & =\frac{u *}{\kappa} \ln \left(10 / z_{0}\right)
\end{aligned}
$$

The median value found is $C_{D_{N 10}}=1.12 \times 10^{-3}$, which is smaller by $7 \%$ than the known $1.2 \times 10^{-3}$ midwind range value (Large \& Pond, 1981), as shown in Figure 10a. Our data present an intermediate behavior when compared to the parameterizations of Smith (1988) and Edson et al. (2013), as shown in Figure 10b. Note that B15-2 data are not taken into account here because they just add scatter compared to the other data.

From the second degree fit to data in Figure 10a, one may even consider the drag coefficient to be rather constant in winds ranging from 4 to $7 \mathrm{~m} / \mathrm{s}$. At wind speeds larger than $7 \mathrm{~m} / \mathrm{s}, C_{D_{N 10}}$ data tend to increase, which is known. However, the number of available points at wind speeds larger than $8 \mathrm{~m} / \mathrm{s}$ is insufficient to propose a reliable parameterization. At wind speeds smaller than $4 \mathrm{~m} / \mathrm{s}$, although the scatter is large, we note that $C_{D_{N 10}}$ estimates visually tend to increase when wind decreases (black dots in Figure 10a). However, it is likely that part of the increase of $C_{D_{N 10}}$ at low winds may be attributed either to the imperfect motion correction as shown in section 3.1 or to the fact that the problem is ill-posed in these conditions (section 1.2.2). Indeed, the proposed fits to data in Figure 10a (plain and dashed gray lines) hardly increase at low wind speeds, which is more obvious with the smoothed version of the parameterization (dashed gray line). With this 

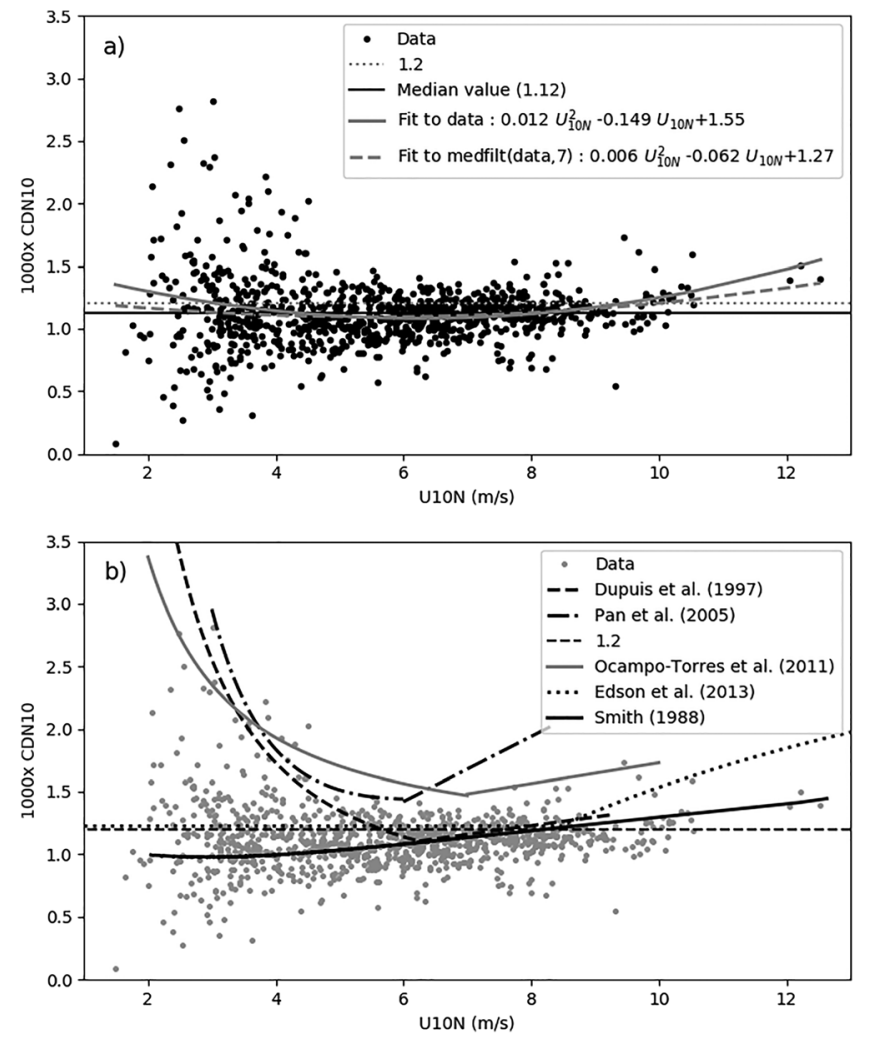

Figure 10. Neutral drag coefficient as a function of the 10-m neutral wind speed (panel a). In the legend, the mention medfilt means that a median low-frequency pass filter over seven points was applied through the data points. In panel b, EC data (gray dots) are compared to several parameterizations of the literature that do not explicitly account for wave characteristics.

last parameterization the resulting bulk estimates of $u *$ compare well to EC and ID $u_{*}$ estimates (not shown) in rms difference, that is, $1.98 \mathrm{~cm} / \mathrm{s}$ (bulk - EC) and $1.27 \mathrm{~cm} / \mathrm{s}$ (bulk - ID), in terms of mean deviation (0.39 and $-0.11 \mathrm{~cm} / \mathrm{s}$, respectively), and in slopes of linear fit to data, 0.96 (bulk - EC) and 1.03 (bulk - ID). Therefore, our data do not confirm the increase in $C_{D_{10 N}}$ at low wind speed present in Dupuis et al. (1997), Pan et al. (2005), or Ocampo-Torres et al. (2011), as shown in Figure 10b. Overall, our data compare best to the parameterization of Edson et al. (2013) in light winds, while in medium-range wind speeds, the historical Smith (1988) parameterization has the best fit to our data.

Note that most of existing wave-dependent parameterizations such as Oost et al. (2002) or Taylor and Yelland (2001) apply to nonfully developed seas, thus not to the swell conditions that correspond to our data set. Consistently with the fact that our data sets correspond to swell cases, the drag coefficient does not clearly scale with inverse wave age $\left(u_{*}\right)$ Csw) for most data (the correlation coefficient is 0.13 or 0.27 in linear or logarithmic coordinates, respectively), although a visual correlation may be suspected, as shown in Figure 11. As in Edson et al. (2013), we find a linear relation between $U_{N 10}$ and $u_{*} / C s w$ (Figure 12a), but please note that it may be here the result of an implicit relation between $U_{N 10}$ and $u *$.

Comparison of our data to the data of Edson et al. (2013) in terms of nondimensional roughness length $z_{0} / \sigma_{\eta}$ as a function of inverse wave age further reveals the particularity of our data set, as shown in Figure 12b. Specifically, the model of Edson et al. (2013) fits well some of the data, but the values of $z_{0} / \sigma_{\eta}$ are scattered and larger than the Edson model for the core of our data. In addition, the model of Edson et al. (2013) has a better fit to our data in terms of Charnock number as a function of inverse wave age than the models of Oost et al. (2002) or Drennan et al. (2003), as shown in Figure 12c. Our estimates of the Charnock coefficient $\alpha$ were calculated as

$$
\alpha=\left(z_{0}-0.11 \nu / u_{*}\right) g / u_{*}^{2}
$$

where $z_{0}$ and $u_{*}$ were EC estimates and $\nu$ was the kinematic viscosity of air taken equal to $1.4510^{-5} \mathrm{~m}^{2} / \mathrm{s}$. As shown in Figure 12d, $\alpha$ is not increasing as a function of $U_{N 10}$ as opposed to what is expected from the parameterization of Edson et al. (2013), which again is possibly specific to our data. Nevertheless, the calculation

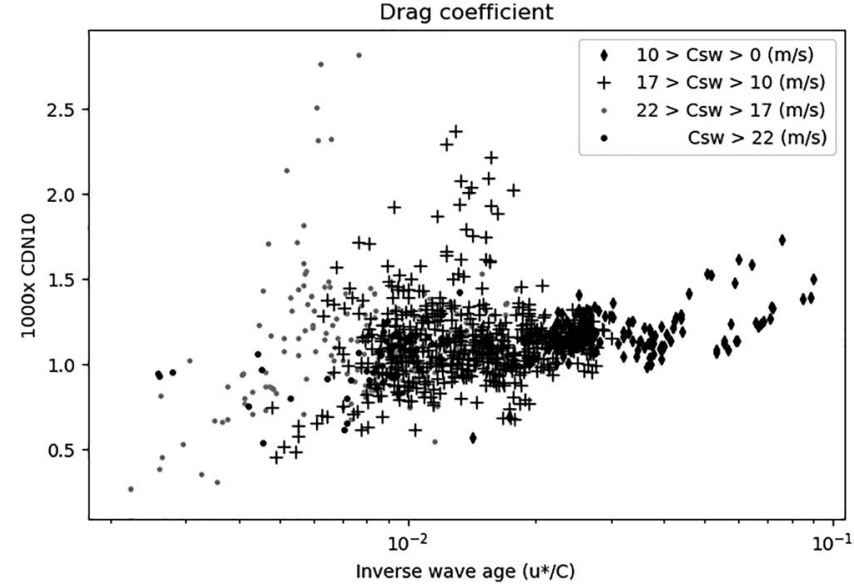

Figure 11. Drag coefficient as a function of inverse wave age and phase speed of the most significant waves. of the median value of the Charnock coefficient gives $\alpha=0.014$ (Figure 12d), which is not too different from the 0.011 reference value in light to medium winds.

\subsubsection{Stanton Number}

As it was not possible to directly calculate EC estimates of $C_{H}$, we used EC buoyancy fluxes combined with bulk latent heat flux values to calculate $C_{H}$ as follows (see Stull, 1988, p. 147):

$$
\begin{gathered}
\overline{w^{\prime} \theta^{\prime}}{ }_{\text {est }}=\left(\overline{w^{\prime} \theta_{v^{\prime}}}{ }_{\mathrm{EC}}-0.61 \bar{\theta} \overline{w^{\prime} q^{\prime}}{ }_{\text {bulk }}\right) /(1+0.61 \bar{q}) \\
C_{H \mathrm{EC}, \text { bulk }}=\overline{w^{\prime} \theta^{\prime}}{ }_{\text {est }} /\left(\overline{U_{10 N}}\left(\overline{S S T}-\overline{T_{10 N}}\right)\right)
\end{gathered}
$$

where $\overline{U_{10 N}}$ and $\overline{T_{10 N}}$ are the 10-m neutral wind and temperature output from the bulk algorithm. Thus, we use true temperature and not potential temperature, which is not an issue as long as potential and true temperatures are not confused in the difference at the denominator of equation (21). However, this calculation may be considered as misleading as 

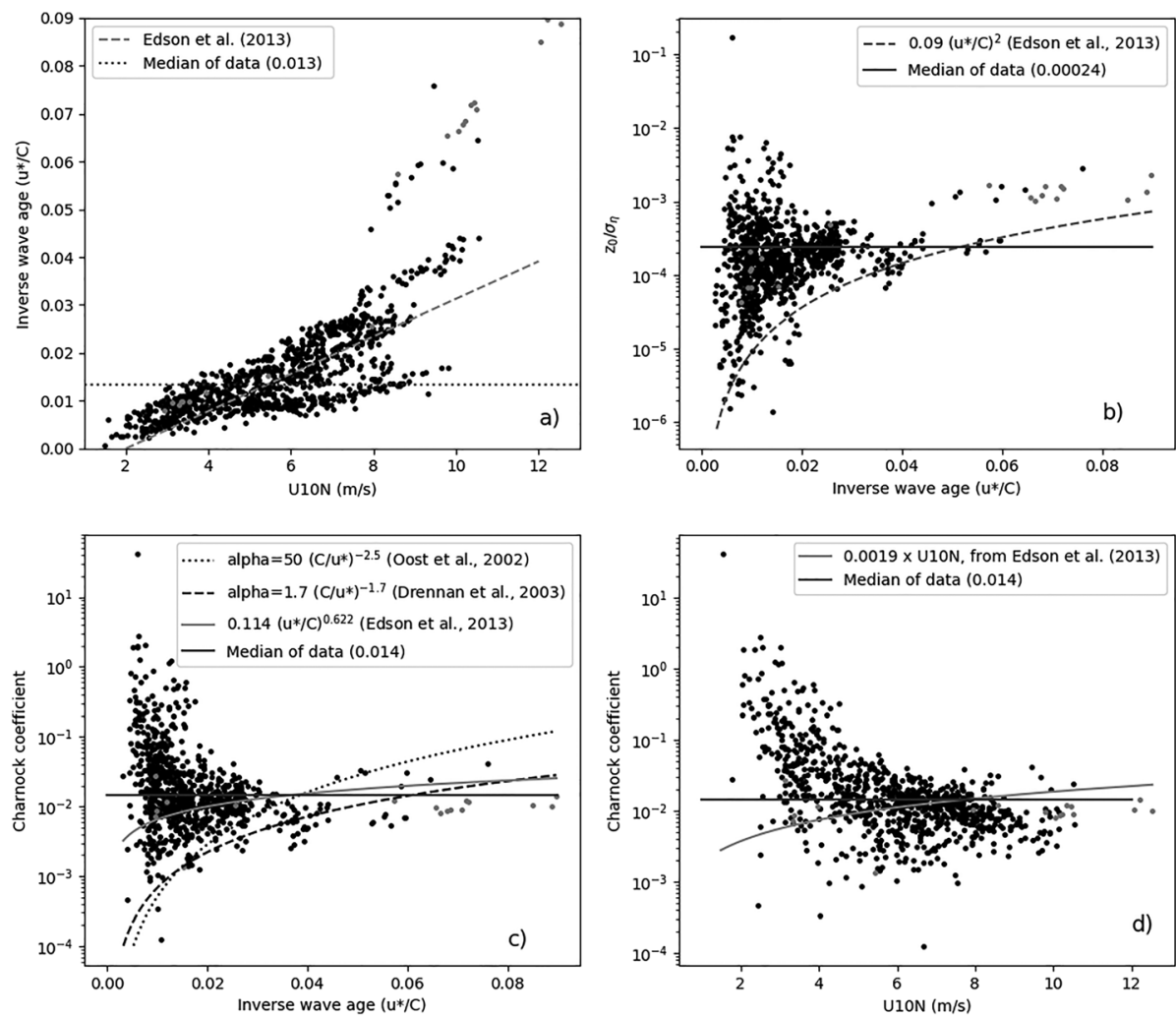

Figure 12. Relation between inverse wave age, wind speed, normalized roughness, and $\alpha$. Dots in gray color denote the U17 short-fetch experiment.

it mixes EC and bulk; thus, we also performed a calculation based on the EC flux only, with the humidity correction as follows:

$$
C_{H V}=\overline{w^{\prime} \theta_{v}^{\prime}} \mathrm{EC} /\left(\overline{U_{10 N}}\left(\overline{\mathrm{SST}_{V}}-\overline{T_{10 V N}}\right)\right)
$$

where only the mean variables $\overline{U_{10 N}}$ and $\overline{T_{10 V N}}$ are outputs from the bulk algorithm. Note that $\overline{\mathrm{SST}_{V}}$ refers to a virtual equivalent of the SST that is calculated using

$$
\overline{\mathrm{SST}_{V}}=\overline{\operatorname{SST}}\left(1+0.61 \overline{q_{S}}\right)
$$

where $\overline{q_{S}}$ is the surface specific humidity output from the bulk algorithm, which mostly depends on SST. In neutral to slightly unstable conditions, referred to as NUC hereafter $\left(-0.1<\frac{z}{L}<0\right), C_{H V}$ is underestimated (median value equal to $\left.0.73 \times 10^{-3}\right)$ compared to the bulk $\left(0.94 \times 10^{-3}\right)$, which is surprising (Figures 13a and 13b). The difference is less marked in unstable conditions $\left(\frac{z}{L}<-0.1\right)$, that is, $0.88 \times 10^{-3}$ for EC data and $0.98 \times 10^{-3}$ for the bulk, respectively. Also, we find that $C_{H V}$ is rather constant or it slightly decreases with wind speed under NUC and unstable conditions, as shown in Figure 13a (black dots and crosses). In neutral to slightly stable conditions, or NSC hereafter $\left(0.1>\frac{z}{L}>0\right)$, the median value of $C_{H V}$ found is $1.14 \times 10^{-3}$, which is again smaller than bulk estimates $\left(1.26 \times 10^{-3}\right)$, but the data have scatter, specifically at low winds (gray dots in Figure 13a). Please note that the $C_{H V}$ estimates peak at unrealistically large values $\left(> \pm 1 \times 10^{-2}\right)$ that are not shown in Figure 13a.

If $C_{H E C \text {, bulk }}$ is plotted as a function of $U_{10 N}$ (Figure 13c), the difference between the median values in NSC $\left(1.20 \times 10^{-3}\right)$ and NUC $\left(0.31 \times 10^{-3}\right)$ is even larger than what was found above for $C_{H V}$ (Figure 13a). The value found in NSC has a good fit to the bulk $\left(1.21 \times 10^{-3}\right.$, Figure 13d), but the scatter is large, such as in Figure 13a (gray dots). As the values found for $C_{H E C \text {,bulk }}$ are largely underestimated in NUC and in unstable conditions, they are doubtful. However, there is no increase of $C_{H E C \text {,bulk }}$ with wind speed, regardless of the stability range considered, as already found for $C_{H V}$. 

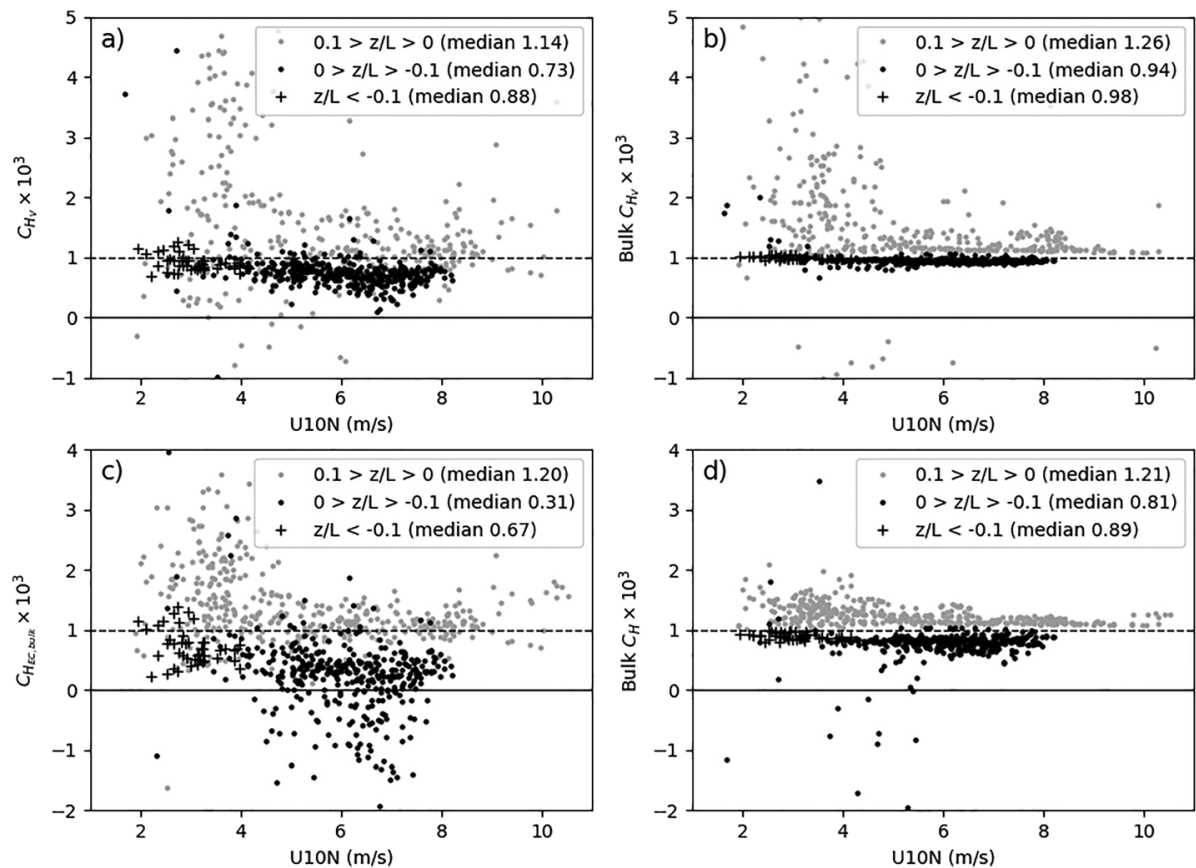

Figure 13. Estimates of the virtual equivalent of the Stanton number (panels $a$ and $b$ ) and of the Stanton number (panels $\mathrm{c}$ and d), as a function of $U_{10 N}$ and $z / L$ range. The estimates were obtained with EC data (panel a), with the bulk algorithm (panels $\mathrm{b}$ and $\mathrm{d}$ ), and with a mixed bulk-EC calculation (panel c). There are outliers not shown at values smaller than $-2 \times 10^{-3}$ and larger than $5 \times 10^{-3}$.

Please note that the warm layer and cool skin effects (e.g., Fairall et al., 2003) were accounted for in the bulk algorithm for obtaining the above results, by using the measured radiation fluxes. Technically, this means that the corresponding parameters so-called jwarm and jcool, respectively, were switched on in the bulk algorithm. Additional tests were performed with the four possible combinations of the jwarm and jcool switches, but this had no major impact on the above results, as reported in Table 7 for the median values found.

\section{Discussion}

\subsection{Imbalance Term}

In section 4.1, we showed that the ID method underestimated $u *$ compared to the EC method. Therefore, there is a lack of energy in the estimates of $u *$ with the ID method. We also found that the value of the

Table 7

Comparison Between Estimates of $C_{H V}, C_{H}$, and $C_{H E C \text {,bulk }}$ Calculated With Different Options in the Bulk Algorithm for Accounting for Warm Layer (jwarm) and Surface Cooling Effects (jcool)

\begin{tabular}{|c|c|c|c|c|c|}
\hline Stanton number $\times 10^{3}$ & Stability & jwarm $=1$ jcool $=1$ & jwarm $=0$ jcool $=0$ & jwarm $=1$ jcool $=0$ & jwarm $=0$ jcool $=1$ \\
\hline \multirow[t]{3}{*}{$C_{H V}$} & unstable & 0.88 & 0.82 & 0.82 & 0.88 \\
\hline & neutral-unstable & 0.73 & 0.69 & 0.70 & 0.73 \\
\hline & neutral-stable & 1.14 & 1.16 & 1.13 & 1.14 \\
\hline \multirow[t]{3}{*}{ Bulk $C_{H V}$} & unstable & 0.98 & 1.14 & 1.14 & 0.98 \\
\hline & neutral-unstable & 0.94 & 1.09 & 1.09 & 0.94 \\
\hline & neutral-stable & 1.26 & 1.13 & 1.12 & 1.24 \\
\hline \multirow[t]{3}{*}{$C_{H E C, \text { bulk }}$} & unstable & 0.67 & 0.51 & 0.51 & 0.67 \\
\hline & neutral-unstable & 0.31 & 0.19 & 0.22 & 0.31 \\
\hline & neutral-stable & 1.20 & 1.19 & 1.17 & 1.19 \\
\hline \multirow[t]{3}{*}{ Bulk $C_{H}$} & unstable & 0.89 & 1.14 & 1.14 & 0.89 \\
\hline & neutral-unstable & 0.81 & 1.10 & 1.10 & 0.80 \\
\hline & neutral-stable & 1.21 & 1.11 & 1.10 & 1.22 \\
\hline
\end{tabular}


Kolmogorov constant was too low $(c=0.48$ instead of 0.55$)$ and that an imbalance term of 0.4 dramatically improved the comparison between ID and EC $u_{*}$ estimates. In addition, we showed that applying a 0.4 constant imbalance term was a better choice than modifying $c$, as already found by B09. However, with a larger data set than the one used in B14, we found that a parameterization of the imbalance term as a function of $\frac{Z}{L}$ had little effect on ID $u *$ estimates. In addition, Cambra (2015) recently found an imbalance term equal to 0.5 for $\mathrm{S} 12$ and A14 data, which is close to the 0.4 value found here.

The origin of the constant term needs to be further addressed. The main differences between OCARINA and research vessel data are in how they move relative to the surface (OCARINA is a wave follower) and the large differences in measurement heights above surface, which are typically on the order of 1 and 15 $\mathrm{m}$, respectively.

First, OCARINA being a wave follower, the sensor is not at a constant height above the mean sea level. However, according to Grare et al. (2013) who compared momentum fluxes in a wind wave tunnel at constant height and with a wave following system, the two measurements result in similar momentum fluxes.

Second, we already explained that OCARINA wind measurements were performed at a height at which the measurements are influenced by the waves (section 1.3). Thus, regarding the ID method, we suspect that the origin of the imbalance term is the pressure-wave term in the TKE equation. This term was not estimated here, because measuring pressure close to the surface is still a challenge.

In order to get around this technical issue, we check hereafter the assumption that $u_{*}$ estimated from EC includes wave effects, whereas $u_{*}$ estimated from ID without imbalance term does not include these wave effects. For this purpose, we applied a high-pass frequency filter to turbulent time series of wind, temperature, and humidity before applying the EC method. Next, we compared the filtered EC $u_{*}$ estimates to ID $u *$ values as a function of the cutoff frequency filter.

We used a normalized cutoff frequency $f_{\text {cut }}=f z / U$, selected in a large range from $10^{-4}$ to $2 \times 10^{-1}$. Next, we converted $f_{\text {cut }}$ values to time period values $T_{\text {cut }}$, and we monitored the evolution of the bias, rms difference, and slope of the comparisons between EC, ID, and bulk estimates of $u_{*}, H_{S V}$, and $L_{E}$, as a function of $T_{\text {cut }}$. As shown in Figure 14 (black line), when filtering the time series of data at periods longer than about $20 \mathrm{~s}$, EC values are in a better agreement with ID values, with smaller rms deviations (in absolute values) and slope of the fit close to unity, compared to what is obtained with larger values of $T_{\text {cut }}$. Note that this is less clear when B15-2 data are accounted for (Gray line in Figure 14). Although the specificity of these data has already been addressed, they are presented here for consistency with Figure $8 \mathrm{~b}$ statistics. At a cutoff value of $8 \mathrm{~s}$, the rms difference exhibits a minimum value, whereas the correlation coefficient is close to its maximum value, which indicates a good agreement between EC and ID $u_{*}$ estimates. As a result, since the cutoff period of $8 \mathrm{~s}$ roughly corresponds to the dominant wave periods of our data set (section 2.3), we show that removing energy in EC $u_{*}$ at the period of the dominant waves contributes to a better agreement between EC and ID $u_{*}$ estimates. Of course, this is subject to caution because the energy that is gradually removed from the EC $u_{*}$ estimates when decreasing values of $T_{\text {cut }}$ also includes energy that possibly results from internal boundary layer processes or intermittency. In spite of these limits, Figure 14 brings an insight to explain that the 0.4 imbalance term is related to waves, namely, to the wind-pressure correlation term, through-for wave modelers-the pressure versus wave slope term also called the form drag (Grare, 2009).

Following the helpful comment of an anonymous reviewer, we decided to further address the issue of the imbalance term by quantifying two Composite versions of the dimensionless Dissipation Rates (CDR hereafter) $\varepsilon \kappa z u_{*}{ }^{-3}$, in which friction velocity was either taken equal to $u_{*} E C$ (with SC motion correction applied) or taken equal to $u_{* \text { bulk }}$ (with the smooth parameterization of drag found in section 4.1 applied). The CDR estimates were then compared to the dimensionless dissipation function $\phi_{U}\left(\frac{z}{L}\right)-\frac{z}{L}-\phi_{\text {imb }}$ with $\phi_{\text {imb }}=0.4$, which simply corresponds to a reorganization of the terms in equation (6). As shown in Figure 15, the CDR values are scattered, markedly close to neutral stability conditions. Nevertheless, the overall median CDR values found are $0.55-0.62$, which indicates consistent values both for the EC and for the bulk estimates of the CDR. The values found are in good agreement with the average value of 0.7 found in near-neutral conditions by Edson and Fairall (1998), Figure 6). 

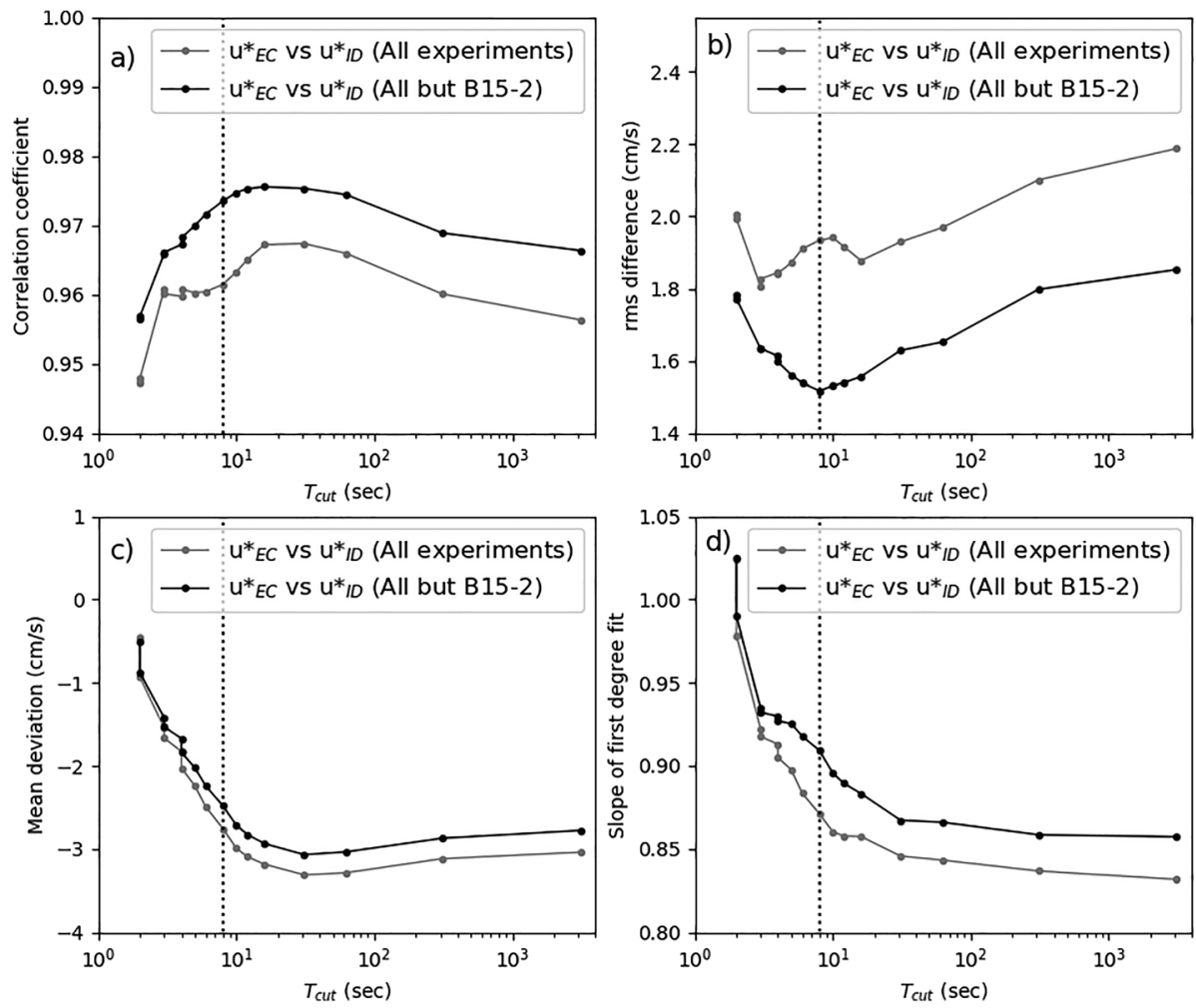

Figure 14. Sensitivity of the statistical elements of the comparisons between ID and EC $u^{*}$ estimates as a function of the cutoff Period ( $\left.T_{\text {cut }}\right)$ of a high-frequency pass filter applied to EC data.

We point here that with a wave-following platform, the EC momentum flux estimates should correspond to a turbulent plus wave-induced flux thus to a total flux, according to Hara and Sullivan (2015, section 5f). In addition, in their simulations, these authors show that at the height of measurement of our platform, $k \zeta \sim 0.1$, the wind profile and the wind shear are hardly modified by the presence of waves (Figure 2 in Hara \& Sullivan, 2015), despite that their simulations correspond to conditions of strong wind forcing. Thus, this also fully justifies that our bulk estimates of momentum flux thus of the drag coefficient are consistent with the corresponding EC estimates, in sections 4.1 and 4.3.1.

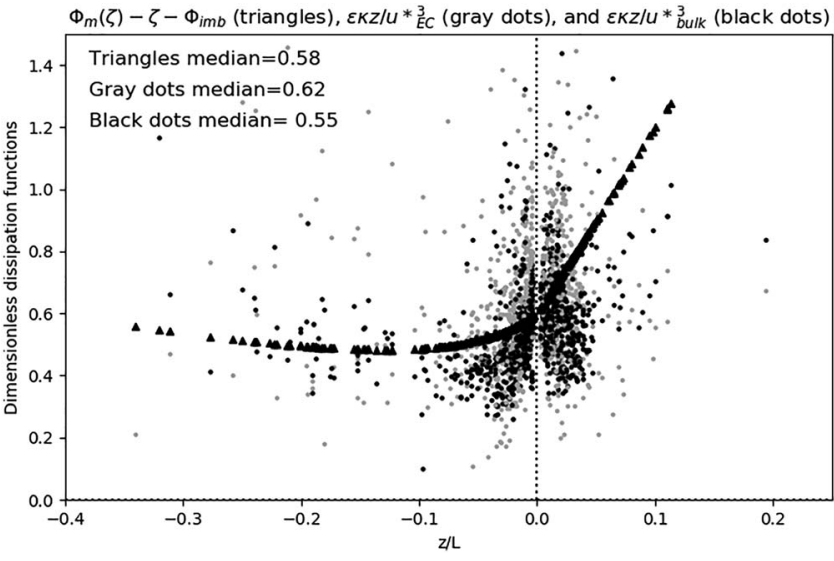

Figure 15. Comparison of the dimensionless dissipation function (triangles) to composite estimates of the dissipation rate that are either normalized with respect to $u_{*} \mathrm{EC}^{3}$ (gray dots) or with respect to $u_{*}$ bulk ${ }^{3}$ (black dots).

\subsection{Spectral Motion Correction Efficiency}

Although the spectral method proposed in section 2.4 for correcting the measured vertical wind component from vertical platform motion was shown to improve spectra and cospectra in sections 2.4 and 3.1.3, it was eventually replaced in section 4.1 with a simple mean correction of the EC $u_{*}$ estimates. As a first justification, we argued there that the changes in the biases were the main outcome from the application of the SC motion correction. It was not possible to better justify this without accounting for the facts that the correction method selected conditioned the value of the ID imbalance term found and the bulk parameterization found for the drag coefficient and that both of which could have a feedback effect on the comparisons between the resulting bulk, ID, and EC $u *$ estimates.

Hereafter, we further assess the efficiency of the methods by comparing the adjusted bulk, EC, and ID $u_{*}$ estimates as obtained (1) with the SC method and (2) with the mean biases used in sections 4.1 and following. Please note that we exclude the scattered B15-2 data, like in section 4.3.1. 

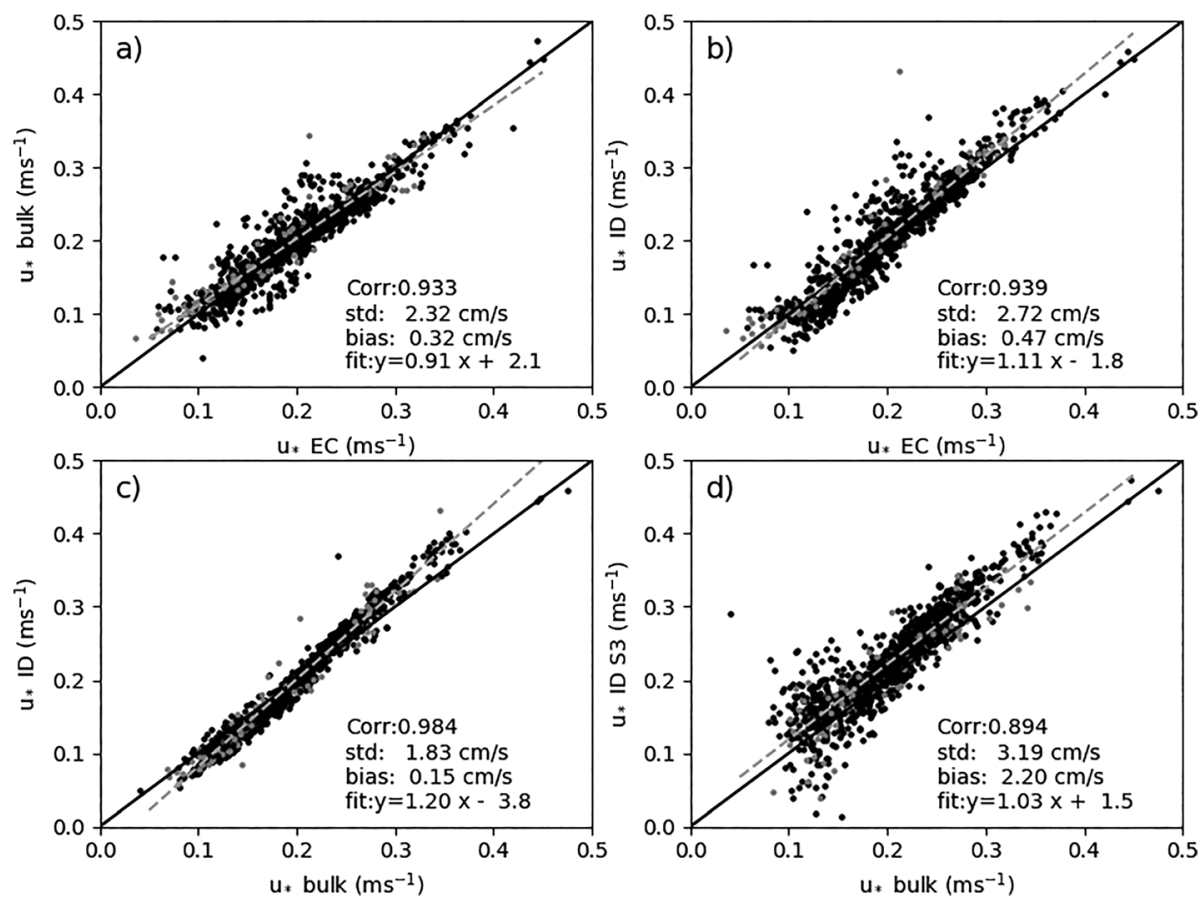

Figure 16. Comparison between SC-corrected EC $u *$ values and the corresponding adjusted ID and bulk estimates of $u *$.

With the SC method applied on individual data, we find $\phi_{\mathrm{imb}}=0.47$, which is slightly larger than the value of 0.4 obtained with the mean biases (section 4.1), and the smoothed parameterization of the $\operatorname{drag}$ is now $C_{D_{N 10}}$ $\mathrm{x} 10^{3}=0.013 U_{10 N^{2}}-0.2 U_{10 N}+2.23$, which may be compared to the smoothed parameterization of Figure 10a. With these parameters, new bulk and ID $u_{*}$ estimates were calculated, next compared to the SC corrected EC $u_{*}$, as shown in Figure 16.
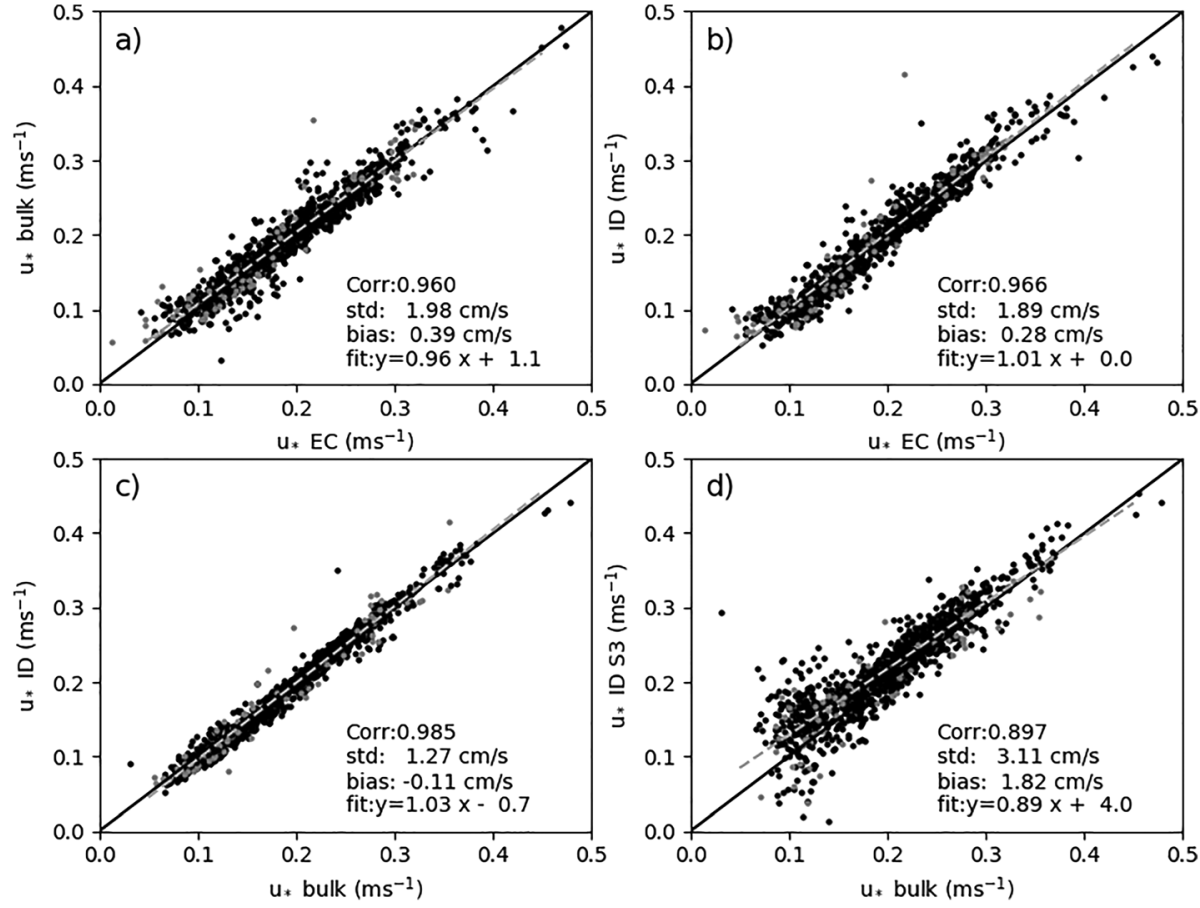

Figure 17. Comparison between $u_{*}$ estimates if the mean motion correction is applied. 
In order to properly assess the respective efficiencies the correction methods, plots comparable to Figure 16 were produced with the mean motion correction, with the parameterization of drag, and with the value of the imbalance term found in section 4.1, as shown in Figure 17. The comparison between Figures 16 and 17 plots clearly indicates that the mean motion correction results in better comparison between bulk, ID, ID S3, and EC estimates of $u_{*}$, specifically in terms of slope of linear fit.

\section{Conclusions}

We analyzed data from a novel-drifting and wave-following platform that was already described in Bourras et al. (2014). It was deployed in four regions during six experiments from 2011 to 2017. The platform recorded $300 \mathrm{hr}$ of data in wave height conditions up to $3 \mathrm{~m}$ and with wind speeds up to $10 \mathrm{~m} / \mathrm{s}$, mostly in the presence of swell. The platform sampled atmospheric turbulence data at 1.5 - $\mathrm{m}$ height, wave characteristics, and water temperature at 10-30 $\mathrm{cm}$ below the surface. A second similar prototype platform was deployed in parallel to the first one during one of the experiments (BBWAVES 2015). The second prototype was equipped with a different sonic anemometer (Campbell EC100) from the sonic anemometer installed on the first prototype (Gill R3-50).

The flux calculation stage was challenging and was specific to the wave-following nature of the platform and to the low height of the turbulence measurements. A spectral method was proposed to correct wind data from platform motion. It was compared to the classical subtraction method. We showed that the spectral method performed better than the subtraction method at frequencies smaller than $0.3 \mathrm{~Hz}$, for the power spectra of the vertical wind component, which was confirmed in a majority of cases by an analysis of the wind cospectra. However, the analysis of the cospectra revealed that a motion correction was not always required, which was the case with $\mathrm{S} 12$ data.

For the two most represented sets of data (S12 and A14), the maximum peaks of the calculated wind cospectra were significantly shifted toward larger values of the normalized frequency $f z / U$ compared to the reference cospectra of Kaimal et al. (1972). Although we have shown that the cospectra were indeed shifted toward larger frequencies under unstable surface boundary layer conditions for S12 data, the observed shifts for S12 and A14 also occur in near-neutral conditions. The shift is therefore attributed to the sea conditions, because our data indicate that the median value of the wave steepness is larger for S12 and A14 data than for the other swell-dominated experiments. We suspect that a frequency scale that would account for wave characteristics would be more appropriate than $f z / U$ for modeling the wind cospectra.

Although the proposed spectral motion correction may be applied on individual data, we have shown that it was more efficient to use it for evaluating mean biases at the scale of an experiment, and next to apply the biases to the noncorrected EC $u *$ values. With this mean motion correction approach, we obtain a good agreement with bulk, EC, and ID $u_{*}$ estimates, that is, rms deviations from 1.3 to $2 \mathrm{~cm} / \mathrm{s}$, and slopes of linear fit close to unity.

A constant imbalance term equal to 0.4 is required in the ID method in order to reconcile the EC and ID $u_{*}$ values. We checked that applying a constant imbalance term is a better option than to modify the value of the Kolmogorov constant. The origin of the imbalance term was analyzed by filtering out the swelldominating periods $(\sim 8 \mathrm{~s})$ from the EC estimates and by estimating the median dimensionless dissipation rate (0.55-0.62), which has a good fit to Edson and Fairall (1998) data in neutral conditions (0.7). Both approaches suggest that the imbalance term found is mainly related to the wave pressure transport term of the TKE budget equation. This is specific to the wave-following nature of our platform and to the fact that the measurements are performed in the WBL, that is, close to the waves. As was well described in Hara and Sullivan (2015), the EC momentum flux estimated with OCARINA data should correspond to a turbulence plus wave-induced flux, thus to a total flux. Close to the surface, the turbulent flux and the dissipation rates are expected to decrease with respect to their wave-induced counterparts, which explains the underestimation of the ID fluxes compared to the EC fluxes if no imbalance is accounted for. Regarding the bulk estimates, Hara and Sullivan (2015) show with large eddy simulations that the profiles of mean wind speed and wind shear are not strongly affected at the height of measurement of our platform, which is an encouraging indication that the bulk estimates of the friction velocity presented in this paper are valid. 
The comparisons presented for the buoyancy flux indicated a good qualitative agreement between bulk, EC, and ID estimates, with rms differences on the order of $3 \mathrm{~W} / \mathrm{m}^{2}$. However, this figure represents a difference of $33 \%$ with respect to the mean absolute value of the flux $\left(9.2 \mathrm{~W} / \mathrm{m}^{2}\right)$, which is large.

Our estimates of the drag coefficient show lower scatter compared to measurements performed on research vessels (e.g., B09). We found a realistic mean value of $1.12 \times 10^{-3}$. Our data have a good fit to the Smith (1988) parameterization as a function of wind speed, although it slightly overestimates it at light winds $(<5 \mathrm{~m} / \mathrm{s})$. The estimates of the drag coefficient are in better agreement with the parameterization of Edson et al. (2013) in light wind conditions. The scatter found in $C_{D_{10 N}}$ estimates is larger at low wind speeds, which is usual. However, we did not find that $C_{D_{10 N}}$ significantly increases at low winds, which is opposed to the behavior of several existing parameterizations such as Dupuis et al. (1997), Pan et al. (2005), or OcampoTorres et al. (2011). In these studies, the apparent increase in surface drag in these parameterizations may be partly attributed to imperfect motion correction or artifacts in data. However, with our EC data the respective contributions of the wave-induced and of the turbulence momentum flux could compensate thus artificially lead to a reduced drag coefficient.

Our data do not show a dependence of the drag coefficient on inverse wave age or phase speed, in average. For the Charnock coefficient, we find a realistic value of 0.014 over the whole range of wind speeds. However, we find that Charnock coefficient is decreasing with wind speed if $U<5 \mathrm{~m} / \mathrm{s}$ and that it is rather constant for larger wind speeds $(5<U<10 \mathrm{~m} / \mathrm{s})$, to which case it is better agreement with the parameterization in COARE 3.0 (Fairall et al., 2003) than with the one in COARE 3.5 (Edson et al., 2013) in which the Charnock coefficient gradually increases with wind speed if $U<18 \mathrm{~m} / \mathrm{s}$. This is possibly specific to our data set, maybe due to the presence of swell.

We calculated Stanton number estimates based on EC buoyancy fluxes corrected with bulk latent heat fluxes. The resulting estimates have uncontrollable biases and scatter, regardless of the calculation method used. The virtual counterpart of the Stanton number $C_{H V}$ was also calculated, as it results from a more straightforward calculation with sonic anemometer data. The values found compare reasonably well to the bulk ones, but they are underestimated by $0.1-0.2 \times 10^{-3}$. Interestingly, Smedman et al. (2007), Figure 17) find that the mean value of $C_{H}\left(0.91 \times 10^{-3}\right)$ is smaller by $0.1-0.18 \times 10^{-3}$ in swell conditions than for younger seas $\left(1.01-1.09 \times 10^{-3}\right)$. This may partly support the underestimation found above, because the bulk estimates of $C_{H V}$ do not explicitly account for wave age. Nevertheless, this parallel with the work by Smedman et al. (2007) would need to be further investigated with new data, because $C_{H}$ and $C_{H V}$ are not the same quantities.

Acknowledgments

The first author would like to thank C. Caudoux and A. Weill (LATMOS laboratory), G. Caulliez, M. Lafont, D. Malengros, and D. Guillemain (MIO

laboratory), O. Ménage and V. Garnier (Ifremer Institute), and P. Antoniotti (subtropic boat rental company) for their help and their technical support during the campaigns. He is grateful to one of the anonymous reviewers who significantly helped us improve the quality of the manuscript. The first author also thanks N. Bourras and M. Libes (MIO) for their valuable computational help. The raw and processed data presented in this article are available at https://doi.org/ 10.17882/59768. The complete AMOP data set is also available at https:// campagnes.flotteoceanographique.fr/ campagnes/14000600/. Efforts are ongoing to make the flux computation code publicly available at https://gitlab osupytheas.fr/. The data and platforms were funded by contracts with IfremerBrest, by the CNRS-INSU LEFE SPURS-STRASSE program, and by the ANR program (ASICS-MED project).
According to our data, $C_{H}$ or $C_{H V}$ do not increase with with wind speed, over a 2-8 m/s range, which was also observed by other authors such as Smedman et al. (2007). It is even rather the opposite; that is, $C_{H V}$ slightly decreases with wind speed.

Back to the science issue raised in section 1, our data suggest that the discrepancy of $10 \%$ found by Brodeau et al. (2017) who compared wind stress from two known bulk algorithms is rather optimistic compared to the achievable accuracy with modern sensors and platforms. Indeed, the 7-10\% confidence on friction velocity found with our data has to be doubled to get its equivalent in terms of wind stress. However, our results would also suggest that several aspects of the relation between wind stress and wave characteristics are yet to be explored, specifically for what regards conditions of swell or swell plus wind-driven waves that are common in the open ocean, although they are less documented with experimental data than fully developed seas in terms of air-sea turbulent momentum exchanges. Large eddy simulations such as the ones performed by Hara and Sullivan (2015) but specific to the swell conditions presented here would be particularly helpful to further analyze the collected data. Independently, these data could further be used to assess the momentum and energy budgets across the interface with respect to wave spectra, following the recent work by Cifuentes-Lorenzen et al. (2018).

\section{References}

Andreas, E. L., Mahrt, L., \& Vickers, D. (2012). New drag relation for aerodynamically rough flow over the ocean. Journal of the Atmospheric Sciences, 69(8), 2520-2537. https://doi.org/10.1175/JAS-D-11-0312.1

Bourras, D., Branger, H., Reverdin, G., Marié, L., Cambra, R., Baggio, L., et al. (2014). A new platform for the determination of air-sea fluxes (OCARINA): Overview and first results. Journal of Atmospheric and Oceanic Technology, 31(5), 1043-1062. https://doi.org/10.1175/ JTECH-D-13-00055.1 
Bourras, D., Weill, A., Caniaux, G., Eymard, L., Bourlès, B., Letourneur, S., et al. (2009). Turbulent air-sea fluxes in the Gulf of Guinea during the AMMA Experiment. Journal of Geophysical Research, 114, C04014. https://doi.org/10.1029/2008JC004951

Brodeau, L., Barnier, B., Gulev, S., \& Woods, C. (2017). Climatologically significant effects of some approximations in the bulk parameterizations of turbulent air-sea fluxes. Journal of Physical Oceanography, 47(1), 5-28. https://doi.org/10.1175/JPO-D-16-0169.1

Cambra, R. (2015). Etude des flux turbulents à l'interface air-mer à partir de données de la plateforme OCARINA (doctoral dissertation). Location: Université de Versailles Saint Quentin en Yvelines.

Charnock, H. (1955). Wind stress on a water surface. Quarterly Journal of the Royal Meteorological Society, 81(350), 639-640. https://doi. org/10.1002/qj.49708135027

Cifuentes-Lorenzen, A., Edson, J. B., \& Zappa, C. J. (2018). Air-sea interaction in the Southern Ocean: Exploring the height of the wave boundary layer at the air-sea interface. Boundary-Layer Meteorology, 169(3), 461-482. https://doi.org/10.1007/s10546-018-0376-0

DeCosmo, J., Katsaros, K. B., Smith, S. D., Anderson, R. J., Oost, W. A., Bumke, K., \& Chadwick, H. (1996). Air-sea exchange of water vapor and sensible heat: The humidity exchange over the sea (HEXOS) results. Journal of Geophysical Research, 101(C5), 12,001-12,016. https://doi.org/10.1029/95JC03796

Donelan, M. A., Drennan, W. M., \& Katsaros, K. B. (1997). The air-sea momentum flux in conditions of wind sea and swell. Journal of Physical Oceanography, 27(10), 2087-2099. https://doi.org/10.1175/1520-0485(1997)027\%3C2087:TASMFI\%3E2.0.CO;2

Drennan, W. M., Graber, H. C., Hauser, D., \& Quentin, C. (2003). On the wave age dependence of wind stress over pure wind seas. Journal of Geophysical Research, 108(C3), 8062. https://doi.org/10.1029/2000JC000715

Dupuis, H., Taylor, P. K., Weill, A., \& Katsaros, K. (1997). Inertial dissipation method applied to derive turbulent fluxes over the ocean during the Surface of the Ocean, Fluxes and Interactions with the Atmosphere/Atlantic Stratocumulus Transition Experiment (SOFIA/ ASTEX) and Structure des Echanges Mer-Atmosphere, Propriétés des Hétérogénéités Océaniques: Recherche Expérimentale (SEMAPHORE) experiments with low to moderate wind speeds. Journal of Geophysical Research, 102(C9), 21,115-21,129. https://doi. org/10.1029/97JC00446

Edson, J. B., \& Fairall, C. W. (1998). Similarity relationships in the marine atmospheric surface layer for terms in the TKE and scalar variance budgets. Journal of the Atmospheric Sciences, 55, 2311-2328. https://doi.org/10.1175/1520-0469(1998)055<2311:SRITMA>2.0 $\mathrm{CO} ; 2$

Edson, J. B., Hinton, A. A., Prada, K. E., Hare, J. E., \& Fairall, C. W. (1998). Direct covariance flux estimates from mobile platforms at sea. Journal of Atmospheric and Oceanic Technology, 15(2), 547-562. https://doi.org/10.1175/1520-0426(1998)015<0547: $\mathrm{DCFEFM}>2.0 . \mathrm{CO} ; 2$

Edson, J. B., Jampana, V., Weller, R. A., Bigorre, S. P., Plueddemann, A. J., Fairall, C. W., et al. (2013). On the exchange of momentum over the open ocean. Journal of Physical Oceanography, 43(8), 1589-1610. https://doi.org/10.1175/JPO-D-12-0173.1

Fairall, C. W., Bradley, E. F., Hare, J. E., Grachev, A. A., \& Edson, J. B. (2003). Bulk parameterisations of air-sea fluxes: Updates and verification for the COARE algorithm. Journal of Climate, 16(4), 571-591. https://doi.org/10.1175/1520-0442(2003)016\%3C0571:BPOASF\% 3E2.0.CO;2

Garcia-Nava, H., Ocampo-Torres, F. J., \& Hwang, P. A. (2012). On the parameterization of the drag coefficient in mixed seas. Scientia Marina, 76(S1), 177-186. https://doi.org/10.3989/scimar.03615.19F

Grachev, A. A., \& Fairall, C. W. (2001). Upward momentum transfer in the marine boundary layer. Journal of Physical Oceanography, 31(7), 1698-1711. https://doi.org/10.1175/1520-0485(2001)031\%3C1698:UMTITM\%3E2.0.CO;2

Grare, L. (2009). Etude des interactions océan-atmosphère à proximité immédiate de l'interface: Application aux vagues de vent et aux vagues extrêmes (doctoral dissertation). Location: Aix Marseille Université.

Grare, L., Peirson, W. L., Branger, H., Walker, J. W., Giovanangelli, J. P., \& Makin, V. (2013). Growth and dissipation of wind-forced deep water waves. Journal of Fluid Mechanics, 722, 5-50. https://doi.org/10.1017/jfm.2013.88

Hanley, K. E., Belcher, S. E., \& Sullivan, P. P. (2010). A global climatology of wind-wave interaction. Journal of Physical Oceanography, 40(6), 1263-1282. https://doi.org/10.1175/2010JPO4377.1

Hara, T., \& Sullivan, P. P. (2015). Wave boundary layer turbulence over surface waves in a strongly forced condition. Journal of Physical Oceanography, 45(3), 868-883. https://doi.org/10.1175/JPO-D-14-0116.1

Högström, U., Rutgersson, A., Sahlée, E., Smedman, A.-S., Hristov, T. S., Drennan, W. M., \& Kahma, K. K. (2013). Air-sea interaction features in the Baltic Sea and at a Pacific trade-wind site: An inter-comparison study. Boundary-Layer Meteorology, 147(1), 139-163. https://doi.org/10.1007/s10546-012-9776-8

Horst, T. W., Vogt, R., \& Oncley, S. P. (2016). Measurements of flow distortion within the IRGASON integrated sonic anemometer and $\mathrm{CO}_{2} / \mathrm{H}_{2} \mathrm{O}$ gas analyzer. Boundary-Layer Meteorology, 160(1), 1-15. https://doi.org/10.1007/s10546-015-0123-8

Kaimal, J. C., \& Finnigan, J. J. (1994). Atmospheric boundary layer flows, their structure and measurement. New York, USA: Oxford University Press. https://doi.org/10.1002/qj.49712152512

Kaimal, J. C., Wyngaard, J. C., Izumi, Y., \& Coté, O. R. (1972). Spectral characteristics of surface-layer turbulence. Quarterly Journal of the Royal Meteorological Society, 98(417), 563-589. https://doi.org/10.1002/qj.49709841707

Kalogiros, J., \& Wang, Q. (2011). Aircraft observations of sea-surface turbulent fluxes near the California coast. Boundary-Layer Meteorology, 139(2), 283-306. https://doi.org/10.1007/s10546-010-9585-x

Large, W. G., \& Pond, S. (1981). Open ocean momentum flux measurements in moderate to strong winds. Journal of Physical Oceanography, 11(3), 324-336. https://doi.org/10.1175/1520-0485(1981)011\%3C0324:OOMFMI\%3E2.0.CO;2

Large, W. G., \& Pond, S. (1982). Sensible and latent heat flux measurements over the ocean. Journal of Physical Oceanography, 12(5), 464-482. https://doi.org/10.1175/1520-0485(1982)012\%3C0464:SALHFM\%3E2.0.CO;2

Larsen, X. G., Smedman, A. S., \& Högström, U. (2004). Air-sea exchange of sensible heat over the Baltic Sea. Quarterly Journal of the Royal Meteorological Society, 130(597), 519-539. https://doi.org/10.1256/qj.03.11

Makin, V. K., \& Mastenbroek, C. (1996). Impact of waves on air-sea exchange of sensible heat and momentum. Boundary-Layer Meteorology, 79(3), 279-300. https://doi.org/10.1007/BF00119442

Miller, S. D., Hristov, T. S., Edson, J. B., \& Friehe, C. A. (2008). Platform motion effects on measurements of turbulence and air-sea exchange over the open ocean. Journal of Oceanic and Atmospheric Technology, 25(9), 1683-1694. https://doi.org/10.1175/ 2008JTECHO547.1

Mueller, J. A., \& Veron, F. (2010). Bulk formulation of the heat and water vapor fluxes at the air-sea interface, including nonmolecular contributions. Journal of the Atmospheric Sciences, 67(1), 234-247. https://doi.org/10.1175/2009JAS3061.1

Ocampo-Torres, F. J., Garcia-Nava, H., Durazo, R., Osuna, P., Diaz Mendez, G. M., \& Graber, H. C. (2011). The intOA experiment: A study of ocean-atmosphere interactions under moderate to strong offshore winds and opposing swell conditions in the Gulf of Tehuantepec, Mexico. Boundary-Layer Meteorology, 138(3), 433-451. https://doi.org/10.1007/s10546-010-9561-5 
Oost, W. A., Komen, G. V., Jacobs, C. M. J., \& Van Oort, C. (2002). New evidence for a relation between wind stress and wave age from measurements during ASGAMAGE. Boundary-Layer Meteorology, 103(3), 409-438. https://doi.org/10.1023/A:1014913624535

Pan, J., Wang, D. W., \& Hwang, P. A. (2005). A study of wave effects on wind stress over the ocean in a fetch-limited case. Journal of Geophysical Research, 110, C02020. https://doi.org/10.1029/2003JC002258

Pedreros, R., Dardier, G., Dupuis, H., Graber, H. C., Drennan, W. M., Weill, A., et al. (2003). Momentum and heat fluxes via the eddy correlation method on the R/V L'Atalante and an ASIS buoy. Journal of Geophysical Research, 108(C11), 3339. https://doi.org/10.1029/ 2002JC001449

Potter, H. (2015). Swell and the drag coefficient. Ocean Dynamics, 65(3), 375-384. https://doi.org/10.1007/s10236-015-0811-4

Prytherch, J., Yelland, M. J., Brooks, I. M., Tupman, D. J., Pascal, R. W., Moat, B. I., \& Norris, S. J. (2015). Motion-correlated flow distortion and wave-induced biases in air-sea flux measurements from ships. Atmospheric Chemistry and Physics Discussions, 15(11), 15,543-15,570. https://doi.org/10.5194/acpd-15-15543-2015

Sahlée, E., Rutgersson, A., Podgrajsek, E., \& Bergström, H. (2014). Influence from surrounding land on the turbulence measurements above a lake. Boundary-Layer Meteorology, 150(2), 235-258. https://doi.org/10.1007/s10546-013-9868-0

Smedman, A.-S., Högström, U., Sahlée, E., \& Johansson, C. (2007). Critical re-evaluation of the bulk transfer coefficient for sensible heat over the ocean during unstable and neutral conditions. Quarterly Journal of the Royal Meteorological Society, 133(622), 227-250. https:// doi.org/10.1002/qj.6

Smith, S. D. (1988). Coefficients for sea surface wind stress, heat flux, and wind profiles as a function of wind speed and temperature. Journal of Geophysical Research, 93(C12), 15,467-15,472. https://doi.org/10.1029/JC093iC12p15467

Stull, R. B. (1988). An introduction to boundary layer meteorology. Springer, Dordrecht: Kluwer Academic Publishers. https://doi.org/ 10.1007/978-94-009-3027-8

Sullivan, P. P., Edson, J. B., Hristov, T., \& McWilliams, J. C. (2008). Large-eddy simulations and observations of atmospheric marine boundary layers above nonequilibrium surface waves. Journal of the Atmospheric Sciences, 65(4), 1225-1245. https://doi.org/10.1175/ 2007JAS2427.1

Taylor, P. K., \& Yelland, M. J. (2000). On the apparent "Imbalance" term in the turbulent kinetic energy budget. Journal of Atmospheric and Oceanic technology, 17, 82-89. https://doi.org/10.1175/1520-0426(2000)017<0082:OTAITI >2.0.CO;2

Taylor, P. K., \& Yelland, M. J. (2001). The dependence of sea surface roughness on the height and steepness of the waves. Journal of Physical Oceanography, 31, 572-590. https://doi.org/10.1175/1520-0485(2001)031<0572:TDOSSR $>2.0$. CO;2

Vickers, D., Mahrt, L., \& Andreas, E. L. (2013). Estimates of the 10-m neutral sea surface drag coefficient from aircraft eddy-covariance measurements. Journal of Physical Oceanography, 43(2), 301-310. https://doi.org/10.1175/JPO-D-12-0101.1

Yelland, M. J., Taylor, P. K., Consterdine, I. E., \& Smith, M. H. (1994). The use of the inertial dissipation technique for shipboard wind stress determination. Journal of Atmospheric and Oceanic Technology, 11(4), 1093-1108. https://doi.org/10.1175/1520-0426(1994)011\%3C1093: TUOTID\%3E2.0.CO;2

Zhang, J. A., Black, P. G., French, J. R., \& Drennan, W. M. (2008). First direct measurements of enthalpy flux in the hurricane boundary layer: The CBLAST results. Geophysical Research Letters, 35, L14813. https://doi.org/10.1029/2008GL034374 